\title{
Ein Rollenmodell zur Einbindung der Endkunden in eine smarte Energiewelt
}

\author{
Lutz Hillemacher • Kai Hufendiek • Valentin Bertsch • \\ Holger Wiechmann • Jan Gratenau • Patrick Jochem • \\ Wolf Fichtner
}

Zusammenfassung Der erfolgreiche Ausbau der Erneuerbaren Energien (EE) bedingt einen Wandel im Energieversorgungssystem. Zum einen müssen Lösungen gefunden werden, wie trotz der hohen Volatilität der EEEinspeisung grundsätzlich die Systemstabilität gewährleistet werden kann, zum anderen müssen Differenzen zwischen Einspeisung und Entnahme, die zunehmend auch auf regionaler Ebene zu beobachten sind, auch regional über den Markt ausgeglichen werden. Hierzu sind neue technische Steuerungs- und modifizierte Marktmechanismen zu entwickeln. Dabei kommt nicht zuletzt auf Grund der politisch vorgegebenen Entflechtung der beteiligten Akteure ihrer konkreten Rollendefinition eine besondere Bedeutung zu. In der Vergangenheit wurde der Rolle der Endkunden allerdings meist eine eher untergeordnete Bedeutung beigemessen. Für ein Gelingen der Umgestaltung des Energieversorgungssystems ist die Einbeziehung der Endkunden jedoch unabdingbare Voraussetzung. Im Rahmen des Forschungsprojektes „MeRegio“ konnte nachgewiesen werden, dass die aktive Einbeziehung der Endkunden in das anreizbasierte Last- und Erzeugungsmanagement erhebliche Potenziale zum Lastausgleich aufweist. Somit kann die stär-

L. Hillemacher $(\bowtie) \cdot$ V. Bertsch $\cdot$ P. Jochem $\cdot$ W. Fichtner Institut für Industriebetriebslehre und Industrielle Produktion, Lehrstuhl für Energiewirtschaft, (KIT) Karlsruher Institut für Technologie, Hertzstraße 16, 76187 Karlsruhe, Deutschland e-mail: lutz.hillemacher@kit.edu

\section{K. Hufendiek}

EnBW Energie Baden-Württemberg AG, Durlacher Allee 93, 76131 Karlsruhe, Deutschland

e-mail: k.hufendiek@enbw.com

H. Wiechmann · J. Gratenau

EnBW Vertrieb GmbH, Schelmenwasenstraße 15, 70567 Stuttgart, Deutschland kere Fokussierung auf den Endkunden einen Beitrag zur Bewältigung obiger Herausforderungen beim Umbau des Energieversorgungssystems leisten.

\section{Definition and Allocation of Roles for Integrating End-Consumers into A Smart Energy World}

Abstract The successful expansion of renewable energies requires a phase of change in the energy supply system. On the one hand solutions have to be found to ensure the system dependability in spite of the high volatility of the feeding-in from renewable sources. On the other hand the differences between feeding-in and demand, which also occurs on the regional level evermore, have to be equalized on the regional level, too. For this purpose, it is necessary to develop new control and modified market mechanisms. The role definition of the involved actors gets an increasing relevance because of the politically predetermined unbundling. However, only a slight attention was paid to the role of the final consumers in the past. For a successful rebuilding of the energy supply system it is nevertheless important to involve the consumers in this process. It could be demonstrated within the research project "MeRegio", that the integration of the consumers into an incentive based demand side management can tap significant potentials to equalize differences between feeding-in and demand. Therefore, the focusing on the final consumers can have an important contribution to rebuild the energy supply system.

\section{Einleitung und Ausgangssituation}

Der Wandel in der Energieversorgung, hervorgerufen durch den erfolgreichen Ausbau der Erneuerbaren Energien (EE), bedingt zur weiteren Versorgungssicherheit eine signifikante 
und nachhaltige Umgestaltung des Energieversorgungssystems, wenn alle Möglichkeiten zur Lösung der Herausforderungen genutzt werden sollen. Um diese Umgestaltung zu realisieren, sind insbesondere die Marktgestaltung und Regulierungsansätze neu zu überdenken bzw. zu erweitern, um Raum für neue technische Ansätze und ökonomische Anreize zu schaffen. Dies soll im Rahmen dieses Artikels erörtert werden.

Dabei müssen im System zwei Ebenen unterschieden werden:

1. Gesamtsystemebene: Hier besteht die Herausforderung darin, wie im Hinblick auf die Systemstabilität mit der hohen Volatilität der EE-Einspeisung aus Wind- und Solarenergie umgegangen werden kann und wie die damit einhergehenden Engpässe im Übertragungsnetz vermieden werden können (Nestle 2007; Droste-Franke et al. 2012).

2. Regionale Ebene: Hier bestehen räumlich differenzierte Herausforderungen durch zeitweise erhebliche Ungleichgewichte von Einspeisung und Entnahme, die bereits heute auf Verteilnetzebene erheblich veränderte Netzzustände einschließlich kritischer Engpasssituationen hervorrufen, die dann auch regional/lokal zu lösen sind (Brauner et al. 2006).

Beide Ebenen sind verknüpft und es besteht bereits heute Handlungsbedarf, der sich zukünftig durch einen weiter gesteigerten Anteil an Erneuerbaren Energien noch verschärfen wird.

Diese Herausforderungen lassen sich - speziell auch auf der regionalen Ebene - nur ökonomisch sinnvoll lösen, wenn alle Möglichkeiten und Eingriffsmöglichkeiten ausgeschöpft werden. Besonders dezentrale Lösungsmöglichkeiten bieten hier den Vorteil einer Wirksamkeit sowohl auf regionaler als auch Gesamtsystemebene. Die auf regionaler Ebene zur Verfügung stehenden Maßnahmen bauen vielfach auf einer intelligenten Verknüpfung von Angebot und Nachfrage auf. Insbesondere hier kommt auch der Einbindung der Endkunden - sozusagen als dritte Ebene - eine neue unabdingbare Rolle zu.

Die derzeitigen Märkte und Mechanismen der Elektrizitätswirtschaft in Deutschland scheinen allerdings keine geeigneten Steuerungswirkungen zu entfalten, da sie einerseits die benötigte Leistungsvorhaltung und -verfügbarkeit nicht ausreichend bewerten und andererseits auch die regionale/lokale Komponente nicht hinreichend reflektieren. Insofern ist ein Überdenken der Mechanismen im gesamten Energieversorgungssystem notwendig - speziell auch im Hinblick auf geeignete Ansätze zur Integration der Flexibilität der Endkunden. Dies wird aktuell im Rahmen der Ansätze zu Smart Grid/Market (BNetzA 2011) bzw. Demand Side Management diskutiert. Im Rahmen dieses Beitrages soll hierfür ein konkreter Ausgestaltungsvorschlag unterbreitet werden, der im Rahmen eines Forschungsprojekts bereits implementiert und erprobt wurde.

Ein entsprechend zu gestaltendes System muss dabei die Aktivierung der notwendigen Maßnahmen und Potenziale ermöglichen. Hierzu ist die Einbindung möglichst aller Stakeholder sowohl auf zentraler als auch regionaler Ebene erfolgsrelevant. Klassisch werden in der Energiewirtschaft dabei die Erzeuger, Netzbetreiber, Politik und Gesellschaft betrachtet. Die Endkunden fanden bei der bisherigen Marktgestaltung nur eine relativ geringe Beachtung, obwohl seit der Liberalisierung des Strom- und Gasmarkts im Jahr 1998 der Einfluss dieser Gruppe in erheblichem Maß gestiegen ist und Nachfragereaktionen generell für das Funktionieren von Energiemärkten von entscheidender Bedeutung sind. Durch den technologischen Fortschritt im Bereich der Informations- und Kommunikationstechnologie (IKT), sowie der dezentralen Erzeugung und Speicherung sind die Aktivitätsmöglichkeiten dieser Gruppe in den letzten Jahren erheblich gestiegen. Da die intelligente Verknüpfung von Erzeugung und Nachfrage einen wesentlichen Schlüssel beim Umbau des Energieversorgungssystems darstellt, sofern sich die Investitionen in einen Netzausbau und entsprechende Reservekapazitäten in volkswirtschaftlich vertretbaren Grenzen halten sollen, ist die Bereitschaft der Kunden, hierbei mitzuwirken, erfolgskritisch.

Diese hängt wiederum wesentlich von der Ausgestaltung der Anreizsysteme ab. Ein mögliches Konzept ist ein dynamischer Preisanreiz, bei dem Nachfrager und (dezentrale) Erzeuger vollständig lokal über die Realisierung ihrer Lastverlagerungspotenziale entscheiden (Nabe et al. 2010). Die Herausforderung aus Sicht der Energielogistik ist hierbei die Prognose der Kundenreaktion auf das Preissignal und daraus resultierend die Bestimmung des optimalen Signalverlaufes.

Eine andere Herangehensweise zur Vernetzung von Erzeugung und Nachfrage stellt ein dynamisches Regelsignal bei einem statischen Preissystem dar. Durch dieses Regelsignal werden mittels Steuerboxen automatisierte Schaltvorgänge über eine intelligente Abfragelogik bei relevanten elektrischen Verbrauchern ohne nennenswerte Komforteinbußen für den Kunden von zentraler Stelle aus vorgenommen. Dem aus Sicht der Energielogistik verlässlichen und feiner ausregelbaren, weil gezielt steuerbaren, Ansatz könnte aus Sicht des Kunden allerdings ein mögliches Akzeptanzproblem gegenüber stehen, da seine Entscheidungsfreiheit zumindest teilweise eingeschränkt wird. Um diesem Akzeptanzproblem begegnen zu können, müssen die Kunden entsprechend kommunikativ eingebunden werden.

Erste Feldtests zur aktiven Einbeziehung und anreizbasierten Steuerung der Endkunden in ein zukünftiges Energieversorgungssystem laufen beispielsweise im Rahmen des 
Tab. 1 Wesentliche Marktakteure und ihre Ziele

\begin{tabular}{|c|c|}
\hline Marktakteure/Stakeholder & Wesentliche Ziele/Kriterien \\
\hline $\begin{array}{l}\text { Erzeuger (von zentralen Groß-KW bis zu } \\
\text { dezentralen Kleinanlagen) }\end{array}$ & $\begin{array}{l}\text { Wirtschaftlichkeit von Investitionen, hohe Deckungsbeiträge, Investitionssicherheit, } \\
\text { langfristige Sicherung von Marktanteilen, teilweise Interesse an Eigenerzeugungs- bzw. } \\
\text { Eigenverbrauchslogiken }\end{array}$ \\
\hline $\begin{array}{l}\text { Verbraucher/Kunden (private Haushalte, Industrie, } \\
\text { Gewerbe, Handel, Dienstleistungen) }\end{array}$ & $\begin{array}{l}\text { Komfort, Versorgungssicherheit, günstige Stromtarife, Wirtschaftlichkeit der eigenen } \\
\text { Produktion (Industrie), Nachhaltigkeit/Ökologie, Akzeptanz, teilweise Interesse an } \\
\text { Eigenerzeugungs- bzw. Eigenverbrauchslogiken (s. o.) }\end{array}$ \\
\hline Energiehandel & $\begin{array}{l}\text { Befriedigung von Angebot und Nachfrage durch Markträumung, Marktliquidität, } \\
\text { Risikomanagement, transparente Referenzpreissignale, länderübergreifende } \\
\text { Harmonisierung der Marktregeln, Regulierungskonformität }\end{array}$ \\
\hline Lieferant oder zukünftig auch Energiedienstleister & $\begin{array}{l}\text { Freier Kundenzugang, Möglichkeit durch wettbewerbliche Produkte Mehrwert zu erzielen, } \\
\text { Kundenzufriedenheit und -bindung, Möglichkeit durch Innovation Wettbewerbsvorteile zu } \\
\text { erzielen, möglichst günstige Strombeschaffung und geringer Bedarf (Energieeffizienz) für } \\
\text { Kunden auf Verbraucherseite bzw. möglichst hohe Preise und hohe (EE-) } \\
\text { Produktionsmengen bei Kunden (aus Sicht des Dienstleisters) auf Erzeugungsseite }\end{array}$ \\
\hline Netzbetreiber (Übertragungs-/Verteilnetz) & Stabiler Systembetrieb, Regulierungskonformität, niedrige Kosten \\
\hline Regulierungsbehörden & $\begin{array}{l}\text { Niedrige Netznutzungsentgelte, Unterbindung von Marktmacht, Schaffen von } \\
\text { diskriminierungsfreiem Marktzugang, Gewährleistung von Rahmenbedingungen zur } \\
\text { Versorgungssicherheit }\end{array}$ \\
\hline Gesellschaft/Politik & Versorgungssicherheit und Nachhaltigkeit zu niedrigen Kosten \\
\hline
\end{tabular}

Forschungsprojektes MeRegio ${ }^{1}$ als Teil der vom Bundesministerium für Wirtschaft und Technologie der Bundesrepublik Deutschland getragenen E-Energy-Projektfamilie. Wesentlicher Bestandteil dieses Projektes waren ein Feldtest sowohl hinsichtlich eines dynamischen Preisanreizes als auch des Einsatzes von Regelsignalen (Hillemacher et al. 2011). Die ersten Auswertungen der gemessenen Verbrauchsdaten der am „MeRegio“-Feldtest teilnehmenden Testkunden zeigen eine signifikante Reaktion auf die Aussendung der Preissignale. Unter Berücksichtigung der Tatsache, dass im Rahmen der frühen Testphase eine ausschließlich manuelle Reaktion der Testkunden auf die ausgesandten Preissignale möglich war und dennoch deutliche Reaktionen auf die Preissignale nachgewiesen werden konnten, ist bei einer zukünftigen automatisierten Gerätesteuerung von zusätzlich realisierbarem Lastverlagerungspotenzial auszugehen.

Der Artikel diskutiert daher zunächst die Voraussetzung für die Aktivierung der kundenseitigen Potenziale: Marktdesign und Regulierungsansätze sowie eine Rollenverteilung zwischen Wettbewerb und reguliertem Bereich mit verschiedenen zugrundeliegenden Regelmechanismen sind die Basis, um ein solches System in Betrieb zu nehmen. Ausgehend davon wird aufgezeigt, welche Marktmechanismen implementiert werden sollten, um dieses Potenzial erschließbar zu machen und die anstehende Energiewende dabei zu unterstützen, eine wettbewerbliche, finanzierbare, nachhaltige

\footnotetext{
${ }^{1}$ MeRegio: Aufbruch zu Minimum Emission Regions - www.meregio. de.
}

und weiterhin versorgungssichere Elektrizitätswirtschaft zu garantieren.

\section{Marktakteure und deren Ziele}

Für die Gewährleistung stabiler Betriebsbedingungen ergeben sich in einem Elektrizitätsversorgungssystem, das in zunehmendem Maße auf fluktuierender Einspeisung basiert, neue Herausforderungen in allen Bereichen des Systems d. h. sowohl in den Wettbewerbsbereichen als auch im regulierten Bereich/Netzbetrieb.

Auf der Wettbewerbsseite werden insb. Maßnahmen und Technologien zum Ausgleich von Erzeugung und Verbrauch stark an Bedeutung gewinnen. Flexibel steuerbare Erzeugung, teilweise dezentral, geeignete Energiespeichertechnologien bzw. moderne IKT und marktbasierte Ansätze zur Umsetzung von „Demand Side Management“ (DSM) Maßnahmen spielen dabei eine zentrale Rolle. Alternativ kommen der Ausbau von Reservekapazitäten oder - allerdings nur auf Gesamtsystemebene - ein verstärkter Stromimport bzw. -export (verbunden mit einer teilweisen Aufgabe des Autarkiegedankens) in Betracht. Im regulierten Bereich wird ein teilweiser Ausbau von Transport- bzw. Verteilnetzen und eine Netzengpassbewirtschaftung auch unterhalb der Transportnetzebene erforderlich.

Neben der stark steigenden Fluktuation auf Erzeugungsseite führt der EE-Ausbau (zumindest in Teilen) zu einer zunehmenden Dezentralisierung des Elektrizitätsversorgungssystems - u. a. da sich EE-Anlagen im Vergleich zu konventionellen Kraftwerken durch kleinere Anlagengrößen auszeichnen. Einhergehend mit der Dezentralisierung ist die 
Anzahl an Akteuren/Stakeholdern im Markt bereits heute erheblich gestiegen und es ist von einer weiter steigenden Anzahl auszugehen, woraus sich zusätzliche Herausforderungen ergeben. Da die verschiedenen Akteure unterschiedliche - oft entgegengesetzte - Ziele verfolgen bzw. verschiedenen Aspekten eine unterschiedliche Bedeutung beimessen (siehe Tab. 1), reduziert sich mit steigender Zahl der Akteure die Planbarkeit im operativen Bereich, aber auch im Hinblick auf die langfristige Entwicklung des Systems. Die Steuerung des Gesamtsystems und ggf. regionaler Teilsysteme wird ungleich komplexer.

Wie aus Tab. 1 hervorgeht, sind neben der Frage der technischen Machbarkeit, der Effektivität oder des Potenzials alternativer Möglichkeiten zur langfristigen Gewährleistung eines sicheren Systembetriebs (z. B. Umsetzung von DSM-Maßnahmen, Kraftwerksausbau, Netzausbau) für die verschiedenen Akteure unterschiedliche Kriterien von besonderer Bedeutung. Diese Stakeholderinteressen sind von hoher Relevanz. Nur wenn sie in hinreichendem Maße für alle Stakeholder gewahrt sind, kann ein stabiles und für alle Akteure akzeptables System entstehen.

Während bspw. für Erzeuger die Frage nach der Wirtschaftlichkeit von Investitionen in Technologien oder Maßnahmen bzw. die Frage nach den mit einer Investition verbundenen, wirtschaftlichen Chancen und Risiken im Vordergrund steht, sind auf Kundenseite u. a. Komfort und Nachhaltigkeit von deutlich höherem Interesse, die ihrerseits wiederum maßgeblichen Einfluss auf die Kundenakzeptanz von Technologien und Maßnahmen haben. Insbesondere bei Netzausbauvorhaben, aber auch bei DSM-Maßnahmen, die einen Einfluss auf die Flexibilität und den Komfort auf Kundenseite haben, gehen subjektive Wahrnehmung und Akzeptanz bei den unterschiedlichen Akteuren weit auseinander.

Eine in Deutschland noch relativ neue Gruppe von Akteuren ist die der Energiedienstleister. Da der Anteil an dezentralen Erzeugungseinheiten jedoch stetig wächst und diese im Besitz oder in Betriebsverantwortung einer Vielzahl vor allem auch kleinerer Anbieter sind, ändern sich die Marktanteile in bisher unbekannter Form und neue Marktteilnehmer haben deutlich leichteren Zugang. Das bisherige Geschäftsmodell der traditionellen Energieversorgungsunternehmen erscheint damit unter Druck und eine Veränderung wird erforderlich. Die Bedeutung von Energiedienstleistungen wird stark zunehmen und es werden neue, innovative Geschäftsmodelle entstehen. Im Fokus der Diskussion um besagten Umbau von Energieversorgern bzw. -lieferanten hin zu Energiedienstleistern stehen dabei bislang Energieeffizienzdienstleistungen. Grundsätzlich sind darüber hinaus aber weit mehr Dienstleistungen denkbar bspw. im Bereich der Optimierung dezentraler Erzeugungsanlagen. Weiterhin wird es in einer dezentralen Energiewelt einen Bedarf geben, Bündelungsfunktionen bei der Anbindung von Endkunden an den Markt zu übernehmen. U. a. können Aufgaben des Bilanzkreismanagements als Dienstleistung oder durch Bündelung einer Vielzahl von Haushalten sowie dezentralen Erzeugern Systemdienstleistungen an Verteilnetzbetreiber angeboten werden (vgl. Abschn. 3). Je nach Kundengruppe, haben die Energiedienstleister dabei unterschiedliche Ziele zu verfolgen, um ihre Kunden optimal zu beraten.

Da für alle Stakeholder die eigene Entscheidungs- und Gestaltungsfreiheit ein wichtiger Aspekt ist, kommt bei der Bewertung alternativer Möglichkeiten zur Gestaltung des künftigen Elektrizitätsversorgungssystems multikriteriellen, partizipativen Ansätzen eine wachsende Bedeutung zu (Bose et al. 1997; Bayley und French 2008). Dabei sind neben den rein ökonomischen Kriterien auch technische, ökologische und sozio-psychologische Aspekte sowie die Einbindung verschiedener Akteure in Entscheidungs- bzw. Bewertungsprozesse zu beachten (Bertsch und Geldermann 2008; Oberschmidt 2010). Methoden aus dem Bereich der multikriteriellen Entscheidungsunterstützung (engl. ,Multicriteria Decision Analysis“ - MCDA) können hier einen wesentlichen Beitrag zur Strukturierung und transparenten Gestaltung von Entscheidungs- und Bewertungsprozessen leisten (von Winterfeldt und Edwards 1986; Belton und Stewart 2002).

Hieraus ergeben sich wiederum Anforderungen an die Rollenverteilung im künftigen Elektrizitätsversorgungssystem. Nach $\$ 12$ EnWG ist die Gewährleistung eines sicheren Systembetriebs Aufgabe der Übertragungsnetzbetreiber (ÜNB), also dem regulierten Bereich zugeordnet. Bestehen zunehmend Gefahren für einen stabilen Netzbetrieb auf regionaler Ebene, fallen auch den Verteilnetzbetreibern (VNB) entsprechende Aufgaben zu. Die Netzbetreiber haben durch entsprechende Eingriffe sicherzustellen, dass es zu keinem Versorgungsausfall kommt. Dabei sind diesen Eingriffen enge Grenzen gesetzt und umgekehrt bleibt offen, welche Eingriffe in den Wettbewerb bereits vorab präventiv vorgenommen werden können, um keine kritischen Netzsituationen entstehen zu lassen.

Allerdings ist schon das Entstehen dieser Frage bezeichnend für die derzeitige Situation im System. Offensichtlich sind die Strukturen aktuell so gesetzt, dass nicht alle Akteure immer einen Anreiz haben, systemstabilisierend zu agieren. Dies ist weniger eine Folge der Liberalisierung als der derzeitigen Systemstrukturen. Um das System nachhaltig zu stabilisieren, sollten also Strukturen geschaffen werden, die allen Akteuren entsprechende Anreize setzen, im Wettbewerb systemstabilisierend zu wirken. Dies würde einerseits das Auftreten kritischer Situation reduzieren und andererseits die Lösung wieder dem Innovationspotenzial des Wettbewerbsmarkts überlassen. Es erfordert jedoch eine grundlegende Veränderung der Systemstrukturen, um die geeigneten Anreize zu setzen.

Dieses Grundverständnis geht auch aus dem in einem Eckpunktepapier der Bundesnetzagentur dargelegten Mo- 
Abb. 1 Prinzip der „Netzampel“ (Hufendiek und Wiechmann 2012)

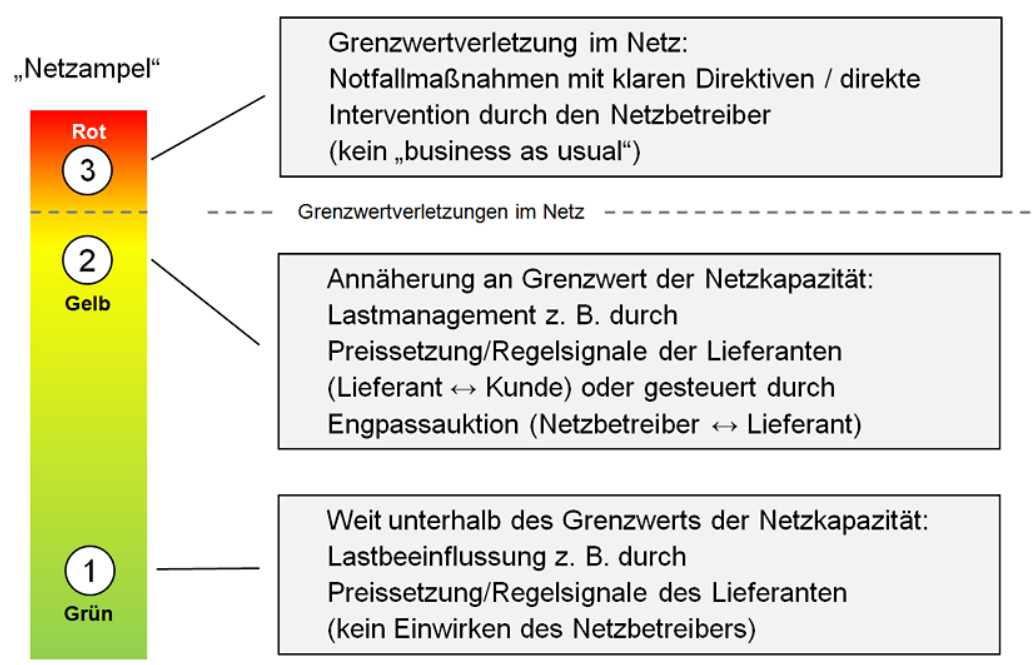

dell hervor (BNetzA 2011). Das Modell ist jedoch noch weiter auszugestalten. Im Zuge der Dezentralisierung ist bspw. zu berücksichtigen, dass der Einfluss der Kunden erheblich ansteigen wird - insb. in einem System, in dem DSMMaßnahmen, dezentrale Erzeugung und Speicherung einen Beitrag zum sicheren Betrieb leisten sollen. Beachtet man die Interessenlage der Kunden, so ist schnell festzustellen, dass eine einfache „Fernsteuerung“ im Rahmen des Lastmanagements kaum auf Akzeptanz stoßen wird. Durch intelligente Lösungen wird das zentrale Kundeninteresse, das sich im Interesse an einer lokalen Optimierung des eigenen Systems und Umfelds manifestiert, berücksichtigt werden müssen.

Auf diese Weise entsteht eine Vielzahl kleinster Energiesysteme bei den Kunden, die sich unabhängig voneinander und hinsichtlich vom Kunden vorgegebener ZielgröBen und Randbedingungen optimieren. Das Erreichen der vielen lokalen Minima stellt jedoch nicht das Erreichen eines Optimums für das Gesamtsystem sicher. Insofern wird ein Steuerungsansatz benötigt, der über eine Verknüpfung der einzelnen Akteure (Kunden) und entsprechende Anreizsetzung bzw. Marktmechanismen ermöglicht, dass ein Optimum auch für das Gesamtsystem erreicht werden kann.

\section{Entwicklung eines Rollenmodells}

Wird das System aus der Perspektive der Systemstabilität und Netzsicherheit betrachtet, so lassen sich prinzipiell zwei Zustände unterscheiden: Entweder das System kann stabil betrieben werden oder es ist unmittelbar kritisch. Im ersten Fall hat der Netzbetreiber ggf. durch geeignete Schaltmaßnahmen oder andere Steuereingriffe im Netz sicherzustellen, dass die Erzeuger, Lieferanten und Abnehmer im Netz ihre Lieferungen ungestört und diskriminierungsfrei abwickeln können. Im letzteren Fall sind entsprechende Notfallmaßnahmen durch den Netzbetreiber - entweder auf Gesamtsystemebene oder auf regionaler Ebene - zu treffen.

Solche Notfallmaßnahmen sind i. d. R. mit Einschränkungen für die Akteure verbunden, sei es die einfache Abschaltung einer Erzeugung bis hin zum Lastabwurf in einzelnen Netzgebieten. Insofern ist es Aufgabe des Netzbetreibers solche Zustände zu vermeiden. Dies kann durch präventive, steuernde Eingriffe erfolgen. Kommen solche Zustände häufiger vor, so werden auch die Eingriffe häufiger, die ihrerseits die Marktmechanismen im Wettbewerbsbereich zumindest stören. Im Extremfall führte es dazu, dass der Netzbetreiber alle Maßnahmen direkt steuern müsste, ein wettbewerblicher Markt wäre damit beendet. Ein anderer Ansatz ist, die Markt- und Systemstrukturen so anzupassen, dass wettbewerbliche Mechanismen vorhanden sind, die das Entstehen von kritischen Situationen systemimmanent vermeiden (siehe „Netzampel“ Abb. 1). Um die dezentralen Potenziale im Bereich der Kunden aktivieren zu können, sind daher entsprechende Strukturen zu schaffen. Dies kann beispielsweise durch die zwei folgenden Mechanismen erreicht werden:

- Auf Gesamtsystemebene erfolgt ein Ausgleich von Angebot und Nachfrage permanent am Markt, inklusive Intraday-Markt. Zusätzlich gibt es Märkte für Systemdienstleistungen, die den darüber hinaus gehenden Bedarf an Flexibilität decken können. Die Kunden müssen daher direkt oder indirekt mit den bereits auf Gesamtsystemebene vorhandenen Märkten verbunden werden können. Dies bringt in dem Gesamtmarkt eine erhöhte Preiselastizität der Nachfrage, die sich positiv auf das Funktionieren des Markts auswirkt (Ockenfels und Crampton 2011).

- Auf regionaler Ebene können entweder analog der Gesamtebene entsprechende Märkte ausgeprägt werden oder vom VNB entsprechende Anreizsignale zur Verfügung gestellt werden, die ein systemstabilisierendes Verhalten 
Abb. 2 Mögliche Aufgabe eines Dienstleisters: Dynamischer Bilanzkreisbewirtschafter (DBB) (Hufendiek und Wiechmann 2012)

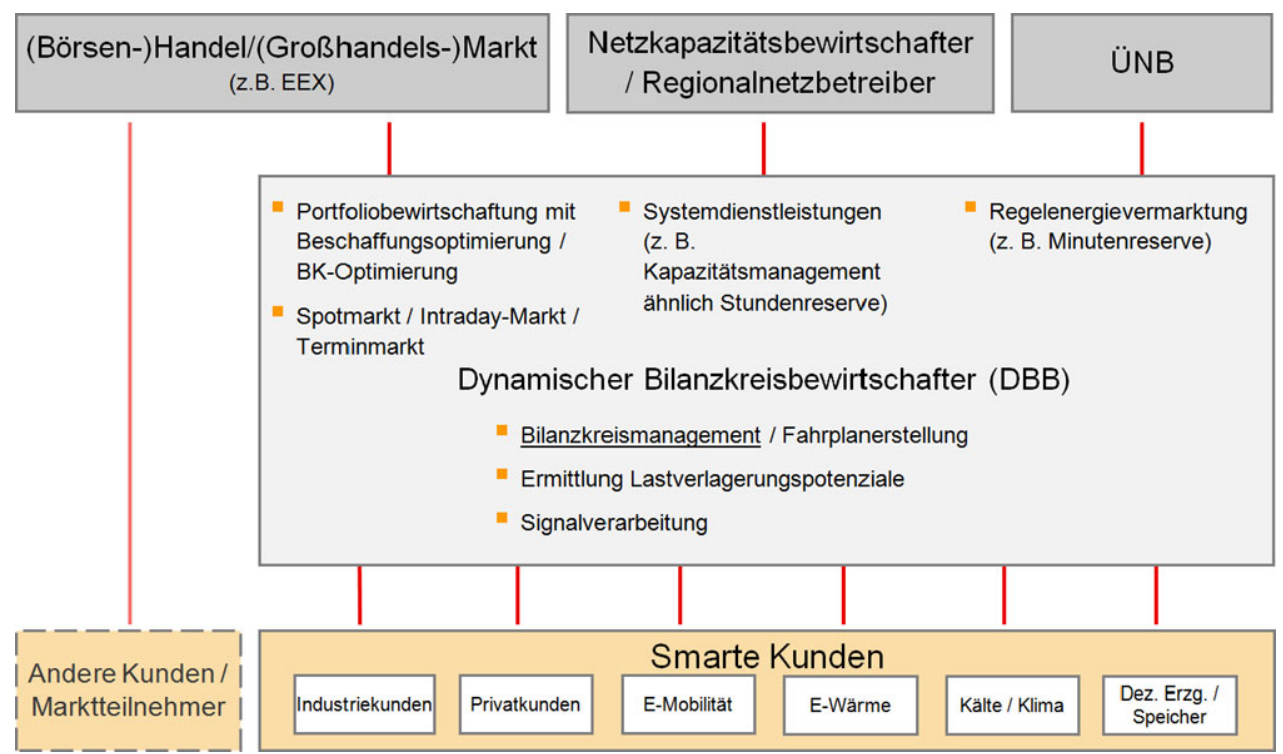

belohnen. Dies könnte entweder durch eine Verbindung der Kunden mit diesen Märkten anhand finanzieller Anreize oder mit Hilfe eines Regelsignals erreicht werden.

Neben der Betrachtung der Systemstabilität und Netzsicherheit kommt den Regeln der Bilanzkreisführung eine zentrale Rolle zu. Im Rahmen der Bilanzkreisführung bewirtschaften sog. Bilanzkreisverantwortliche (BKV) ihre Bilanzkreise, indem sie die an Kunden zu liefernden Strommengen so kaufen bzw. die aus ihren Kraftwerken produzierten Strommengen so verkaufen, dass die ge- bzw. verkauften Mengen stets ausgeglichen sind, d. h. die Bilanzkreise eine ausgeglichene Bilanz je Viertelstunde aufweisen. Die Funktion des Bilanzkreisverantwortlichen ist mithin Bestandteil der wettbewerblichen Seite und nur die Bilanzkreiskoordination und -abrechnung erfolgt durch den regelzonenverantwortlichen Netzbetreiber. Bis zum heutigen Tag ist dieses Geschäft vergleichsweise einfach zu betreiben, weil ein meist thermisch geprägter Kraftwerkspark so eingesetzt werden kann, dass er die prognostizierten Stromabsatzmengen erzeugen kann („Erzeugung“ folgt „Verbrauch“). Da die Kunden in der Regel über statische Stromtarife verfügen und es faktisch keine automatisiert steuerbare Lasten gibt, ${ }^{2}$ ist aus Sicht der Bilanzkreisverantwortlichen die Prognose der Nachfrage mit einem vergleichsweise geringen Aufwand verbunden. Im Zuge der Energiewende mit ihrem Ausbau an nicht steuerbaren, fluktuierenden Einspeisungen aus Erneuerbaren Energien (Wind, Photovoltaik) verändert sich dieses Prinzip grundlegend. Künftig muss sich

\footnotetext{
${ }^{2} \mathrm{Da}$ die unterbrechbaren Verbrauchseinrichtungen wie Speicherheizungen oder Wärmepumpen über temperaturabhängige Standardlastprofile versorgt werden, stellen diese keine faktisch automatisiert steuerbare Lasten dar. Das Potenzial für eine Automatisierung wäre aber vorhanden.
}

die Nachfrage an der Erzeugung orientieren, weil sich das Stromangebot stärker am Winddargebot und der Sonneneinstrahlung ausrichtet. Hierzu bedarf es dynamischer Ansätze zur Energiebeschaffung, was auch eine erhöhte ,Dynamisierung“, sprich eine gesteigerte Komplexität der Bilanzkreisführung der Bilanzkreisverantwortlichen mit sich bringt. Aus einem „statischen“ Bilanzkreisbewirtschafter wird so ein „Dynamischer Bilanzkreisbewirtschafter“ (DBB), der zusätzliche Aufgaben hinsichtlich Kommunikation zu den Kunden aber auch an der Schnittstelle zum Verteilnetzbetreiber übernehmen kann.

Da die Vielzahl der einzelnen Endkunden sicherlich nicht direkt an den Großhandelsmärkten aktiv sein kann oder möchte, wird hier ein Bedarf für eine Bündelungsfunktion entstehen. Dies ist direkt mit dem Aktienhandel vergleichbar: Kleinere Anleger können nicht direkt an den Wertpapierbörsen handeln, sondern beauftragen Intermediäre, dies für sie zu erledigen. Im Elektrizitätssystem könnte diese Aufgabe der Dynamische Bilanzkreisbewirtschafter übernehmen, der verschiedene Kunden bündelt und seinerseits mit den Großhandelsmärkten und den jeweiligen Netzbetreibern verbunden ist (siehe Abb. 2). Seine Kernaufgabe liegt in einer smarten Energielogistik/Energiebeschaffung und der damit verbundenen Bewirtschaftung des dazugehörigen Bilanzkreises, in dem die Kunden liegen. Darüber hinaus kann der Dynamische Bilanzkreisbewirtschafter - in Analogie zur Vermarktung von Regelenergie aus Kraftwerken - Systemdienstleistungen und Regelenergie den Netzbetreibern aus seinem Kundenportfolio anbieten. Da es sich um eine wettbewerbliche Rolle handelt, stehen mehrere Dynamische Bilanzkreisbewirtschafter im Wettbewerb zueinander. So kann auch ein hohes Maß an Innovationsfähigkeit gewährleistet werden, so dass die Dynamischen Bilanzkreisbewirtschafter den Kunden unterschiedliche Angebote und Services anbieten bzw. zur Verfügung stellen können. 


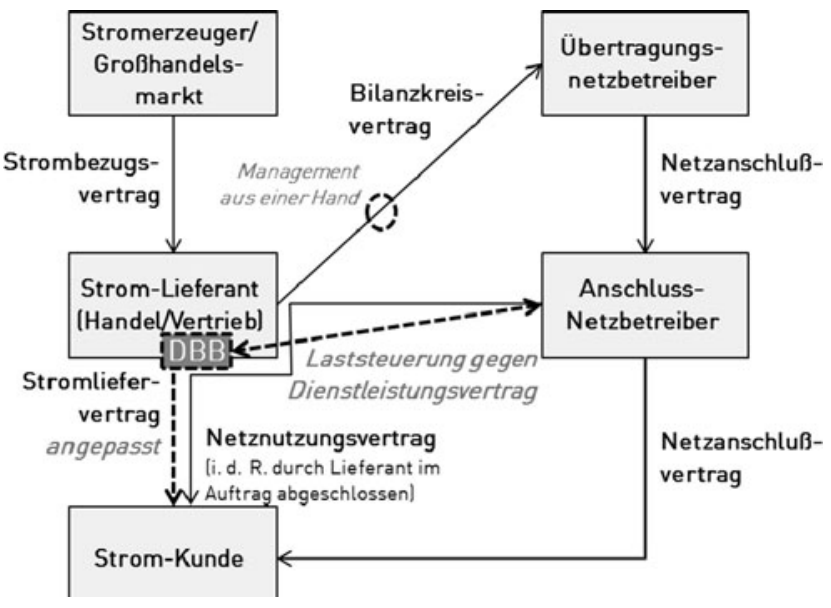

Abb. 3 Vertragsbeziehungen und Rollenmodell (heutige Vertragsbeziehungen in Schwarz dargestellt; vorgeschlagenes Rollenmodell, wobei die Rolle des DBB beim Lieferanten angesiedelt ist, gestrichelte Linien und graue kursive Schrift)

Ein Vorteil des vorgeschlagenen Rollenmodells zeigt sich bei der Betrachtung des heute vorhandenen Rollenmodells und der damit verbundenen vertraglichen Beziehungen bei der Belieferung von Endkunden mit Strom (siehe Abb. 3): Der Lieferant bzw. Händler beliefert den Endkunden mit Strom und schließt üblicherweise in seinem Auftrag gleichzeitig den Netznutzungsvertrag mit dem Anschlussnetzbetreiber ab. Zur Sicherstellung der Belieferung bezieht er Strom von einem Erzeuger oder am Großhandelsmarkt und betreibt hierfür einen Bilanzkreis, für den er einen $\mathrm{Bi}$ lanzkreisvertrag mit dem Übertragungsnetzbetreiber abgeschlossen hat. Aus dem Bilanzkreisvertrag ergibt sich eine Verpflichtung für den Lieferant (Bilanzkreisverantwortlicher) diesen ausgeglichen zu halten.

Die Zuordnung eines Dynamischen Bilanzkreisbewirtschafters gewissermaßen als Element eines Energiedienstleisters in den Wettbewerbsbereich - und nicht in den regulierten Bereich als Teil eines Netzbetreibers - basiert auch auf dem grundsätzlichen Marktrollenverständnis, das in den EU-Richtlinien sowie den nationalen Gesetzen formuliert ist. Auf Basis der Entflechtungsvorgaben und der Tatsache, dass ausschließlich der Marktseite Energiemengenbilanzierungen im Sinne von Kundenbelieferungen zugestanden werden, ist es eben Aufgabe der Marktseite Kundenbeziehungen zur Belieferung mit Energie zu pflegen. Daher werden folgerichtig die Auswirkungen skizziert, wenn die Aufgabe des Dynamischen Bilanzkreisbewirtschafters dem wettbewerblichen Bereich, und hier exemplarisch dem Lieferanten, zugeordnet wird. Abbildung 3 zeigt die Änderungen in den Vertragsbeziehungen schematisch, die dazu im Vergleich zu den heutigen Vertragsbeziehungen notwendig sind.

Im Einzelnen ergeben sich die gestrichelt gezeigten Modifikationen in den Vertragsbeziehungen:
Als zusätzlicher Vertrag muss - falls der AnschlussVerteilnetzbetreiber ein Netzengpassmanagement betreiben will - eine entsprechende Vereinbarung zwischen Lieferant und Verteilnetzbetreiber geschlossen werden, die regelt, zu welchen Konditionen der Dynamische Bilanzkreisbewirtschafter Dienstleistungen (d. h. Laststeuerung) für den Verteilnetzbetreiber betreibt und nach welchen Regeln dies abzuwickeln ist. Alle weiteren Veränderungen betreffen bestehende Vertrags- und Leistungsbeziehungen, die vom Lieferant als Dynamischer Bilanzkreisbewirtschafter konsistent aus einer Hand gesteuert werden können. Technisch kann die Verbindung vom Dynamischen Bilanzkreisbewirtschafter zu den Kunden über entsprechende Signale erfolgen. Dies können sowohl dynamische Preissignale oder auch Regel- bzw. Regelsignale sein:

- Bei Preissignalen wird der Kunde direkt am Marktgeschehen beteiligt. Er kann solche Preissignale einfach in eine bei ihm ablaufende lokale Optimierung seines Energiesystems einbauen (z. B. KWK-, Speicher- oder Eigennutzungsoptimierung sowie Optimierung der Ladevorgänge bei Elektrofahrzeugen). Er kann auch durch verändertes Verhalten - auf freiwilliger Basis - entsprechend reagieren. Die Entscheidungshoheit liegt vollständig beim Kunden.

- Bei sog. Regelsignalen muss der Kunde einer entsprechenden Steuerung durch den Dynamischen Bilanzkreisbewirtschafter zustimmen (z. B. über eine intelligente Abfragelogik). Dann allerdings hat er i. d. R. keinen direkten Einfluss mehr auf die Steuerung. Es ist davon auszugehen, dass er für die Einwilligung vom Dynamischen Bilanzkreisbewirtschafter eine entsprechende Prämie erhält. Für den Dynamischen Bilanzkreisbewirtschafter besteht aber auf diesem Weg eine verbesserte Steuerbarkeit und eine deutlich erhöhte Verlässlichkeit.

Die Wahrnehmung der Aufgabe des Dynamischen Bilanzkreisbewirtschafters von Dienstleistern, also im wettbewerblichen Bereich, bietet einige Vorteile, speziell im Hinblick auf die Konformität mit den derzeit bestehenden Regelungen und Rollen. Ein Anreiz- oder Regelsignal berührt immer die Abnahme des Kunden. Dies hat mehrfache Rückwirkungen, u. a. auf den Lieferanten: Einerseits verändert sich das Gleichgewicht des Bilanzkreises in dem die Abnahmestelle des Kunden angemeldet ist. Hier ist der Lieferant als Bilanzkreisverantwortlicher in der Pflicht, den Bilanzkreis ausgeglichen zu halten. ${ }^{3}$ Andererseits berührt es den

\footnotetext{
${ }^{3}$ Es wird hier angenommen, dass der Lieferant auch die Beschaffungsprozesse bzw. den Börsenhandel selbst durchführt also selber ein Händler ist. In größeren Energieversorgungsunternehmen (EVU) ist es üblich, dass diese Aufgabe durch die separaten Handelsgesellschaften erfolgt. Dort liegt dann die Bilanzkreisverantwortlichkeit. Da die Handelshäuser aber dem Wettbewerbsbereich zugeordnet sind, ändert sich an dem Prinzip der BKV-Zuordnung in dem Wettbewerbsbereich nichts.
} 
Liefervertrag, wenn sich die Abnahmestruktur oder Menge verändert. Insofern ist es nicht möglich, dass ein Dritter steuernd eingreift, ohne mit dem Lieferanten entsprechende Regelungen zu treffen. Übernimmt der Lieferant die Verantwortung für die Aufgabe des Dynamischen Bilanzkreisbewirtschafters, entfallen diese zu treffenden Regelungen.

Die Beeinflussbarkeit der Last entsprechend Vorgaben des Verteilnetzbetreibers ist dadurch in keiner Weise eingeschränkt. Ähnliche Prozesse existieren bereits im Bereich der Systemdienstleistungen auf Gesamtsystemebene: Der Übertragungsnetzbetreiber ruft die nach einen Ausschreibungsverfahren vergebenen vertraglich vereinbarten Systemdienstleistungen bei einem oder mehreren Lieferanten ab. Die Lieferanten entscheiden, aus welchen Anlagen, die zuvor präqualifiziert wurden, die Leistung erbracht wird. Durch Bündelung einer Vielzahl von Endkunden mit entsprechender Informations- und Kommunikationstechnologie kann der Dynamische Bilanzkreisbewirtschafter dem Verteilnetzbetreiber (oder auch dem Übertragungsnetzbetreiber) auf dieselbe Weise Dienstleistungen anbieten. Der Informationsaustausch bzw. das Senden der notwendigen Signale erfolgt dabei dann nicht direkt zwischen Netzbetreiber und Endkunden, sondern zwischen Netzbetreiber und Dynamischem Bilanzkreisbewirtschafter auf der einen Seite sowie zwischen Dynamischem Bilanzkreisbewirtschafter und Endkunden auf der anderen Seite.

Ein solches System, das sowohl ein dynamisches Preissignal als auch ein Regelsignal, ausgehend von einem dynamischen Bilanzkreisbewirtschafter enthält, wurde im Rahmen des Forschungsprojekts MeRegio implementiert.

\section{Das MeRegio-Steuerungssystem}

Das Forschungsprojekt MeRegio war Teil der vom Bundesministerium für Wirtschaft und Technologie geförderten EEnergy-Projektfamilie und wurde in einem Konsortium unter Führung der EnBW und wissenschaftlicher Begleitung durch das Karlsruher Institut für Technologie (KIT) durchgeführt. Ein wesentlicher Aspekt dieses Projektes war der Ausgleich zwischen Stromnachfrage von Endkunden und zentraler wie dezentraler Erzeugung mit Hilfe moderner IKT (MeRegio 2012). Die entwickelten Konzepte wurden dabei sowohl mit Hilfe von Simulationen als auch in einem Feldversuch mit ca. 1.000 Testkunden erprobt (Hirsch et al. 2010).

Im Rahmen des MeRegio-Feldversuches erfolgten die Anreize zur Last- und Erzeugungsverschiebung auf zwei Arten: Zum einen wurde mittels eines Preissignals eine Lastbzw. Erzeugungsverlagerung angeregt, zum anderen wurde ein Regelsignal, eingesetzt, das seinerseits eine kurzfristige Verschiebung des Last- bzw. Erzeugungsverhaltens bewirkte. Die Signale beider Typen hatten jeweils für stündliche Zeitintervalle Gültigkeit.
Die Aussendung von Preissignalen wurde in der Vergangenheit bereits in einigen Feldversuchen erprobt. In der Regel orientierten sich diese Versuche am klassischen Ansatz des Peak-Load-Pricings (Boiteux 1949; Steiner 1957). Als Beispiel für Deutschland sei etwa der Eckernförder Feldversuch genannt, bei dem von 1994 bis 1996 die Testhaushalte ein stetiges Preissignal erhielten, das auf einer am Grenzkostenverlauf der Stromproduktion orientierten Preisfunktion basierte (Morovic et al. 1998). Im Rahmen von MeRegio wurde dieser Ansatz um eine Komponente, die der Verfügbarkeit der dezentralen Erzeugung in der Region Rechnung trägt, ergänzt. Dies gestattet folglich eine regional höher aufgelöste Berücksichtigung der schwankenden Verfügbarkeit des dezentral erzeugten Stromes als es eine ausschließliche Anlehnung an den Ansatz des Peak-Load-Pricings auf Großhandelsmarktebene erlauben würde. Somit können auch auf regionaler Ebene auftretende Engpässe und sonstige reaktionsbedürftige Netzsituationen berücksichtigt und durch die anreizbasierte Verbrauchssteuerung mittels regional differenzierter Preis- und Regelsignale ausgeglichen werden.

Von den ca. 1.000 MeRegio-Testkunden verfügten mit Beginn der dritten Projektphase rund ein Drittel über elektrische Verbraucher (z. B. Gefrierschrank, Geschirrspüler), dezentrale Erzeugungsanlagen (z. B. Mini-Blockheizkraftwerk) oder Stromspeicher (z. B. Batteriesysteme), die über eine mit dem Gerät verbundene Steuerbox automatisiert auf beide Signaltypen reagieren konnten. Die restlichen Testkunden reagierten manuell ausschließlich auf das Preissignal, das über ein im Haushalt befindliches Display oder aber über eine Web-Applikation kommuniziert wurde.

Abbildung 4 gibt einen Überblick über den Ablauf des projektspezifischen Last- und Erzeugungsmanagements. Die Bestimmung der Preissignale wurde jeweils für den Folgetag vorgenommen, so dass die Testkunden Kenntnis über die Bepreisung am frühen Abend des Vortages erhielten. Grundlage für die Generierung der Preissignale waren in der Hauptsache Prognosen des Last- und Erzeugungsverlaufes sowie Kosten der Strombeschaffung, repräsentiert durch die Kurse der europäischen Strombörse EEX. Im Forschungsprojekt MeRegio kam ein dreistufiges Preissignal in stündlicher Auflösung zur Anwendung (siehe Tab. 2). Die Zuordnung der Tarifstufen zu den einzelnen Stunden eines Tages erfolgt dynamisch und wurde den Kunden am Vorabend verbindlich bekannt gegeben.

Die Signalgenerierung wurde mit Hilfe einer für dieses Projekt entwickelten optimierenden Modellfamilie anhand der zuvor genannten Eingangsparameter durchgeführt. Die Eingangsgrößen lassen sich dabei flexibel gewichten. So kann bei der Signalfestlegung dem Ansatz der Großhandelsmarktpreis basierten Tarifierung oder aber der möglichst optimalen Ausnutzung der (regionalen) dezentralen Stromerzeugung eine größere Bedeutung beigemessen werden (Hillemacher et al. 2011). 
Abb. 4 Schematischer Ablauf des Last- und Erzeugungsmanagements des MeRegioFeldversuches
Tab. 2 Struktur des variablen MeRegio-Stromtarifs

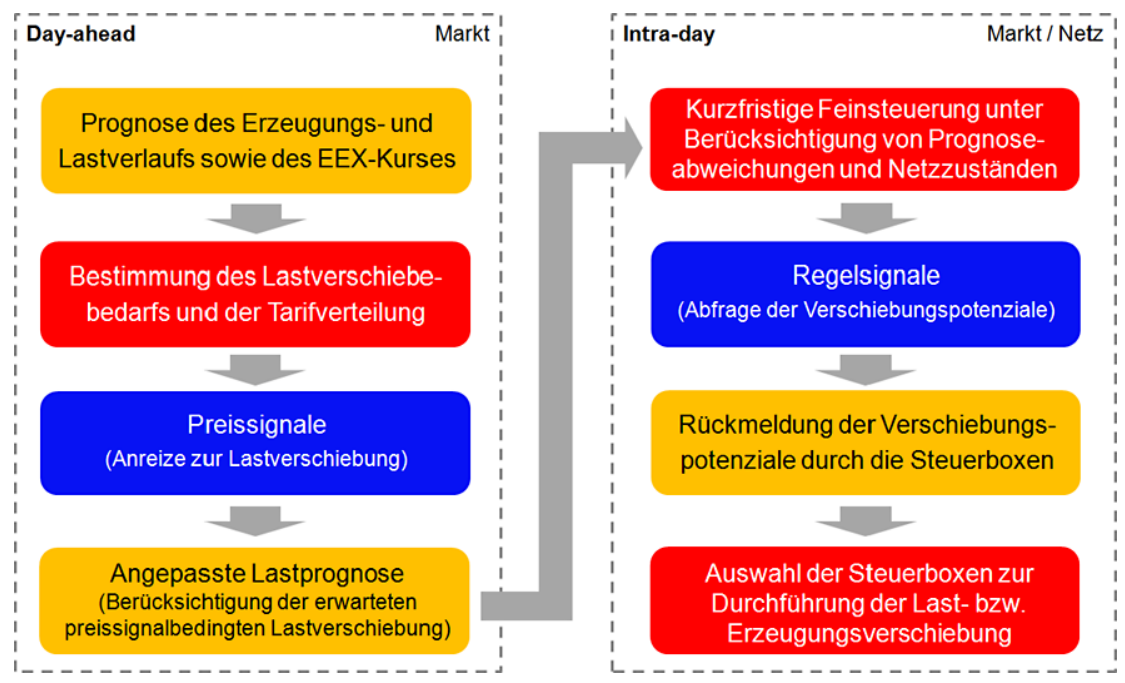

\begin{tabular}{lll}
\hline Tarifstufe & Bepreisung & \\
\cline { 2 - 3 } & 1. November 2009-30. April 2010 & 1. Mai 2010-31. März 2012 \\
\hline Tarifstufe 1 („SNT“) & $15,81 \mathrm{ct} / \mathrm{kWh}$ & $15,00 \mathrm{ct} / \mathrm{kWh}$ \\
Tarifstufe 2 („NT“) & $19,31 \mathrm{ct} / \mathrm{kWh}$ & $20,00 \mathrm{ct} / \mathrm{kWh}$ \\
Tarifstufe 3 („HT“) & $22,31 \mathrm{ct} / \mathrm{kWh}$ & $25,00 \mathrm{ct} / \mathrm{kWh}$ \\
\hline
\end{tabular}

Die Bepreisung der Tarifstufen und damit einhergehend die Tarifspreizung des MeRegio-Feldtests wurden nach Beginn des Feldversuches im November 2009 erstmalig zum Mai 2010 verändert. Über die konkrete Bepreisung der einzelnen Tarifstufen gibt Tab. 2 Auskunft. Zum April 2012 wurde erneut die Tarifierung modifiziert, um die Sensitivität des Verhaltens der Kunden auf geänderte Preise analysieren zu können. Auf Grund der zum Berichtzeitpunkt noch nicht in ausreichendem Maße vorliegenden Messdaten der letzten Projektphase beschränken sich die folgenden Ausführungen jedoch auf den Zeitraum bis zum Jahresende 2011, so dass die zweite Anpassung in die nachfolgenden Auswertungen noch nicht eingeflossen ist. Im Hinblick auf die Validierung der grundsätzlichen Funktionsfähigkeit des vorgeschlagenen Rollenmodells hat der weitere Versuch keine Auswirkungen, wenn gezeigt werden kann, dass Lastveränderungen erreicht werden.

Nach Festlegung der Tarifverteilung für den Folgetag wird sodann eine korrigierte Lastprognose erstellt, die die erwartete Lastverschiebung auf Grund der Preissignale in jedem Zeitintervall berücksichtigt.

Ausgehend von der am Vortag unter Berücksichtigung des Preissignalverlaufes angepassten Lastprognose sowie der Prognose der regionalen Erzeugung erfolgt untertägig ein fortlaufender Abgleich der Messwerte mit den Prognosen des Last- und Erzeugungsverlaufes. Die kurzfristige Feinsteuerung zum Ausgleich von Prognoseabweichungen einerseits aber auch die Realisierung netzseitig erforderli- cher Änderungen im Last- oder Erzeugungsverhalten andererseits geschieht mit Hilfe von Regelsignalen. Unter einem Regelsignal wird im Rahmen von MeRegio ein Signal verstanden, das steuerbare elektrische Verbraucher bzw. regelbare, dezentrale Erzeugungsanlagen über eine mit der Gerätschaft verbundene Steuerbox erhalten. Das Regelsignal gibt dabei die Richtung der beabsichtigten Verhaltensänderung vor (z. B. Last verringern). Eine detaillierte Beschreibung dieses Ablaufes des Last- und Erzeugungsmanagements findet sich in Hillemacher et al. (2012).

\section{Erste Ergebnisse des MeRegio-Feldversuches}

Da der Feldversuch zum Abfassungszeitpunkt gerade erst abgeschlossen ist und die Auswertung hinsichtlich der Reaktion auf die Regelsignale wegen der Einführung erst in der letzten Projektphase noch über das Projektende hinaus läuft, fokussieren sich die folgenden Ergebnisse auf die preissignalbedingten Reaktionen der MeRegio-Testkunden.

$\mathrm{Zu}$ Beginn des Feldversuches wurde überprüft, ob überhaupt eine grundsätzliche Reaktion der Testkunden auf die Preissignale zu beobachten ist. Hierzu wurde - wie auch bei der späteren Detailanalyse - der Lastgang der Versuchsteilnehmer mit dem einer Kontrollgruppe verglichen. Die Auswahl der Kunden für die Referenzgruppe erfolgte im Hinblick auf eine den Testkunden möglichst ähnliche Zusammensetzung, sowohl hinsichtlich des Verbrauchsverhaltens 


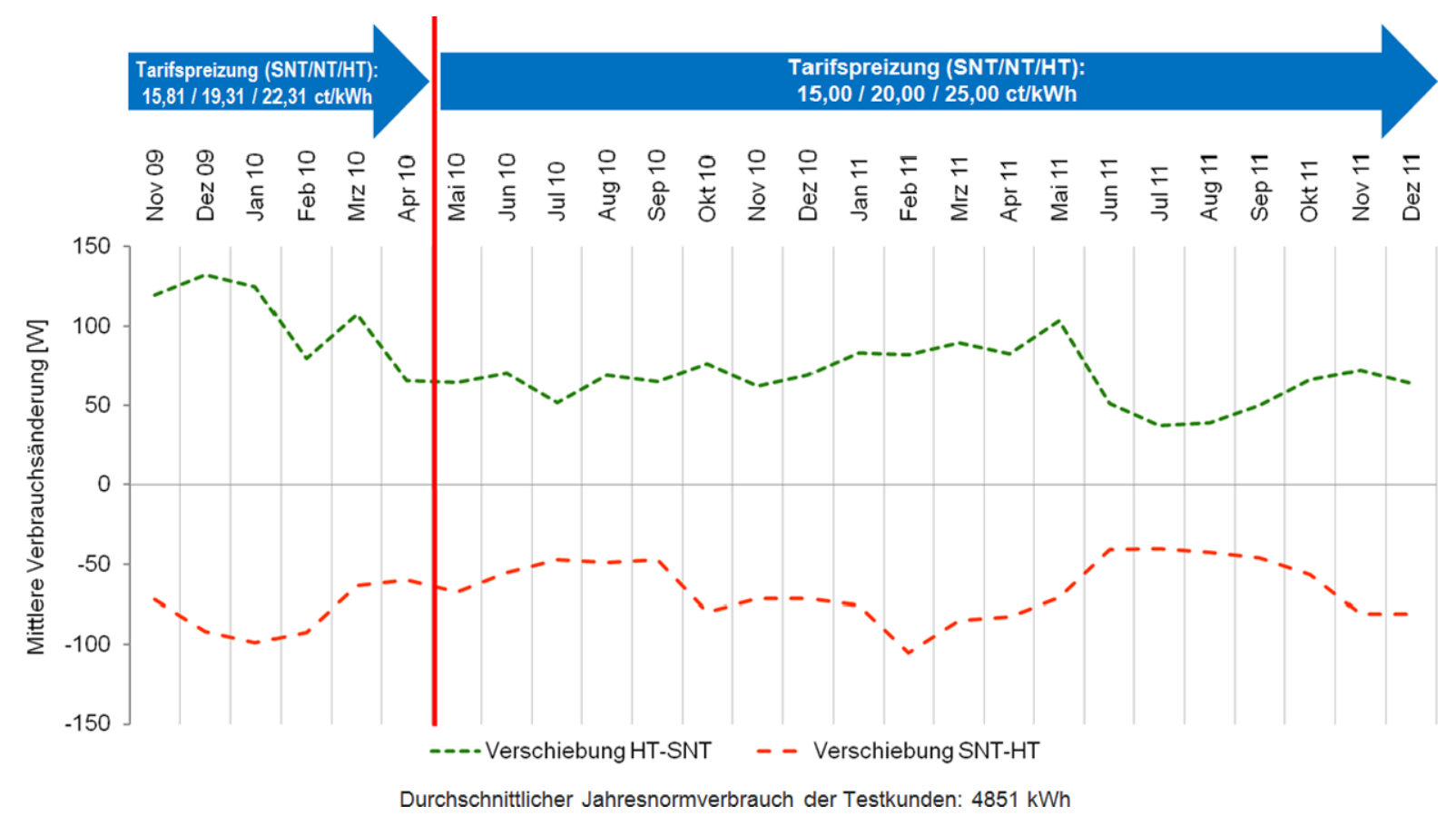

Abb. 5 Mittlere Verbrauchsänderungen der MeRegio-Testkunden gegenüber der Referenzgruppe in Abhängigkeit vom Tarifwechsel

vor Beginn des Versuches als auch der soziodemografischen Durchmischung. Der Vergleich des Verhaltens der Testkunden mit dem der Referenzkunden erlaubt eine weitgehende Elimination externer Einflüsse aus den Messdaten wie z. B. der Witterung. Auf Grund der Freiwilligkeit der Versuchsteilnahme und auf Grund versuchstechnischer Restriktionen ist die Zusammensetzung der Versuchs- und notwendigerweise auch der Kontrollgruppe als nicht repräsentativ in $\mathrm{Be}$ zug auf die Gesamtbevölkerung zu konstatieren, so finden sich in diesen Gruppen überproportional viele 40-60 jährige Eigentümer einer selbstbewohnten Immobilie.

Wiesen vor Beginn des Versuches die Lastgänge beider Kundengruppen eine $95 \%$ ige Korrelation auf, zeigt sich nach Beginn der Aussendung der Preissignale, dass die Abweichungen im Verbrauchsverhalten der Testkunden deutlich vergrößert werden konnten. Mit Hilfe eines Kolmogorov-Smirnov-Tests konnte gezeigt werden, dass die Abweichungen vor und nach Aussendung der Preissignale unterschiedlichen Verteilungen folgen. Somit konnte in einem ersten Schritt eine Reaktion auf die Preissignale angenommen werden.

Daneben war für die Detailanalyse auch von Interesse, ob die Bereitschaft der Testkunden, ihren Stromkonsum an der Tarifverteilung auszurichten, über die Versuchsdauer erhalten blieb oder ob ein nachlassendes Interesse zu beobachten ist. Ein nachlassendes Interesse würde durch die Versuchsausgestaltung der sog. Bestpreis-Abrechnung begünstigt, da auf Grund der vertraglichen Bestimmungen kein Testkunde finanziell gegenüber der Anwendung des u. a. für die Referenzkunden geltenden festen Tarifs schlechter gestellt wird.
Um hierzu Erkenntnisse zu gewinnen, wurde der Mehr- bzw. Minderkonsum der Testkunden gegenüber der Kontrollgruppe bei einem Wechsel von der hochpreisigen auf die günstigste Tarifstufe (HT $\rightarrow$ SNT) bzw. von der günstigsten auf die hochpreisige Tarifstufe (SNT $\rightarrow$ HT) über die Versuchsdauer betrachtet und mittels eines F-Tests auf statistische Signifikanz überprüft. Diese Tarifstufenkombination wurde gewählt, da sich hier erwartungsgemäß die anschaulichsten Veränderungen herauskristallisieren.

Für die ersten 26 Versuchsmonate, also einen Zeitraum von über 2 Jahren, ist der Verlauf in Abb. 5 dargestellt. Bei der Betrachtung der zuvor beschriebenen Kurven fällt auf, dass zu Beginn des Feldtests die Testkunden stärker auf die Tarifstufenwechsel reagieren. Zum Frühjahr 2010 hin stellt sich jedoch ein über einen längeren Zeitraum recht stabiles Niveau ein. Zu berücksichtigen ist dabei auch, dass zu Beginn des Feldtests nur eine relativ geringe Anzahl von Testkunden, nämlich einhundert, zur Verfügung standen, während die Referenzgruppe mit etwa dreihundert, zahlenmäßig deutlich stärker ist. Aus diesem Grund können sich bestimmte Verhaltensweisen bereits einzelner Testkunden (z. B. urlaubsbedingte Abwesenheit) stark auf das Gesamtergebnis auswirken. Gleichwohl würde sich vor diesem Hintergrund ein nachlassendes Interesse bereits weniger Testkunden im Kurvenverlauf sichtbar niederschlagen. Dies kann jedoch nur geringfügig beobachtet werden, so dass das Eintreten von Sättigungseffekten bei den in der Auswertung enthaltenen Versuchsteilnehmern weitgehend zu verneinen ist. 
Tab. 3 Tarifstruktur- und tarifstufenabhängige relative Laständerung der MeRegio-Testkunden im Vergleich zur Referenzgruppe (Betrachtungszeitraum November 2009-Dezember 2011)

\begin{tabular}{lll}
\hline & $\begin{array}{l}\text { Tarifstruktur } \\
\text { bis 30. April 2010 }\end{array}$ & $\begin{array}{l}\text { Tarifstruktur } \\
\text { ab 1. Mai 2010 }\end{array}$ \\
\hline Tarifstufe 1 (SNT) & $5,9 \%$ & $6,2 \%$ \\
Tarifstufe 2 (NT) & $-1,3 \%$ & $-1,6 \%$ \\
Tarifstufe 3 (HT) & $-6,4 \%$ & $-4,4 \%$ \\
\hline
\end{tabular}

Des Weiteren wurde untersucht, wie sich eine veränderte Tarifspreizung, die ab Mai 2010 eingeführt wurde, auf das Kundenverhalten auswirkt. Die neue Spreizung hatte im Vergleich zur zunächst verwendeten eine größere und gleichmäßigere Spreizung über die drei Tarifstufen. Wie aus Tab. 3 ersichtlich ist, verändert sich das Verhalten der Versuchsteilnehmer beim Auftreten der Tarifstufen 1 und 2 nur unwesentlich. Einzig beim Auftreten der hochpreisigen Tarifstufe 3 ergibt sich eine deutlichere Veränderung. Nach Umstellung der Tarifstruktur fällt der Minderverbrauch trotz nun größerer Differenz zu den beiden anderen Tarifstufen um zwei Prozentpunkte geringer aus.

Dabei ist jedoch zu berücksichtigen, dass die Tarifumstellung nach sechs Monaten erfolgte, die zweite Periode aber 20 Monate dauerte. Damit weisen beide Perioden unterschiedliche Zeitdauern auf. Die erste Periode enthielt dadurch ausschließlich Wintermonate, während die zweite Periode die Jahreszeiten gleichmäßiger abdeckt, so dass auch jahreszeitabhängige Effekte eine Rolle spielen könnten (vgl. Abb. 6). Eine Eingewöhnungsphase der Testkunden zu Versuchsbeginn ist mit Blick auf Abb. 3 ebenfalls zu unterstellen und bei der vergleichenden Betrachtung zu bedenken, so dass der Befund hinsichtlich der Unterschiede zwischen den Perioden eher auf diese Effekte zurückzuführen sein sollte.

Die in Tab. 3 angegebenen relativen Verbrauchsänderungen sind über den gesamten Betrachtungszeitraum aggregiert. Naturgemäß variiert das Lastverlagerungspotenzial jedoch saisonal sowie tageszeit- und tagtypabhängig recht stark. Tabelle 4 sowie Abb. 4 und 5 geben einen Überblick über die Schwankungen bei der relativen Lastverlagerung der MeRegio-Testkunden. Aus Gründen der Übersichtlichkeit beschränken sich diese Darstellungen auf die günstigste und teuerste Tarifstufe. Für die Tagesrandlagen sowie die Nachtstunden der Tarifstufe 3 an Wochenenden kann hierbei auf Grund einer zu geringen Anzahl von Messwerten noch keine belastbare Aussage getroffen werden. Einer der Gründe hierfür ist, dass in den ersten beiden Projektphasen ein Auftreten der hochpreisigen Tarifstufe 3 am Wochenende vertriebsseitig von vornherein ausgeschlossen wurde.

Die in Tab. 4 und den Abb. 6 und 7 verwendete Nomenklatur für die Stunden referenziert immer den Beginn des Zeitintervalls, also repräsentiert z. B. die Zeitscheibe „11“ die Stunde von 11-12 Uhr; die Messwerte wurden durch
Abgleich der mittleren saisonalen Tagesverbräuche der Testkunden vor und nach Beginn des Feldtests um einen grundsätzlichen Minderverbrauch, der sich auf Transparenz- und grundsätzliche Einspareffekte zurückführen lässt, bereinigt. Da die hier untersuchten Kunden ausschließlich manuell auf die am Vortag kommunizierte Tarifverteilung reagieren können, sind die doch deutlichen Lastveränderungen umso bemerkenswerter und weisen einerseits auf ein breit vorhandenes Potenzial sowie andererseits auf eine hohe Bereitschaft der Kunden in gewissen Anwendungsbereichen bei entsprechenden Anreizen Verhaltensänderungen vorzunehmen.

Erwartungsgemäß reagieren die Testkunden auf eine Verbilligung ihres Strombezugs, hier dargestellt durch die grünen Linien, tendenziell mit einer Lastzunahme, während bei einer Verteuerung, repräsentiert durch die roten Linien, tendenziell eine Lastabnahme beobachtet werden kann. Allerdings zeigt sich, dass die Ausmaße der Lastverschiebung tageszeitabhängig starken Schwankungen unterliegen.

Wie sich bei einer Betrachtung der Abb. 6 zeigt, ist an Wochentagen das relative Lasterhöhungspotenzial am stärksten vor- und nachmittags ausgeprägt. Dagegen kann in den Morgen- und insbesondere in den Abendstunden nur eine geringe relative Lasterhöhung bei Gültigkeit der günstigsten Tarifstufe festgestellt werden. Besonders im Winter ist in diesen Zeiträumen sogar eine relative Lastverringerung gegeben. Grundsätzlich ist das relative Potenzial zur Lasterhöhung bei den Testkunden mit Ausnahme der Mittagsstunden im Sommer größer als im Winter. Demgegenüber fällt das relative Lastverringerungspotenzial in den Wintermonaten geringfügig größer als im Sommer aus. Hier ist vor allem am Morgen und in den Abendstunden eine deutliche relative Lastabnahme bei einer hochpreisigen Tarifstufe erkennbar. Somit kristallisieren sich hier tageszeitabhängige Hauptrichtungen der Lastverlagerung heraus, was bedeutet, dass sich bestimmte Tageszeiten eher zur Lastverringerung, nicht jedoch zur Lasterhöhung eignen und umgekehrt.

Ein etwas anderes Bild ergibt sich bei Betrachtung der relativen Lastverlagerung an Wochenenden und Feiertagen, dargestellt in Abb. 7. Hier sind die Potenziale deutlich symmetrischer verteilt als wochentags, abgesehen von einer Spitze beim relativen Lasterhöhungspotenzial in den frühen Nachmittagsstunden. Zu berücksichtigen ist jedoch, dass auf Grund der zuvor bereits erwähnten geringeren Anzahl an Messwerten für die Tarifstufe 3 die Betrachtung der Lastabsenkungen auf die Zeit zwischen 6 und 22 Uhr beschränkt bleiben muss. Bedacht werden muss ferner, dass Grundlage der vorstehenden Ausführungen stets das relative Lastverlagerungspotenzial ist. Das absolute Lastverlagerungspotenzial kann auf Grund der tageszeit- und saisontypischen Schwankungen der Stromnachfrage, wie sie sich anhand der typischen Standardlastprofile des Bundesverband der Energie- und Wasserwirtschaft e.V. (BDEW) zeigen, in größerem Maße schwanken und andersartig ausgeprägt sein. 
Tab. 4 Tageszeit- und jahreszeitabhängige Lastveränderung der MeRegio-Testkunden im Vergleich zur Referenzgruppe am Beispiel der Tarifstufen 1 und 3

\begin{tabular}{|c|c|c|c|c|c|c|c|c|}
\hline \multirow[t]{4}{*}{ Stunde } & \multicolumn{8}{|c|}{ Relative Laständerung der MeRegio-Testkunden im Vergleich zur Referenzgruppe } \\
\hline & \multicolumn{4}{|c|}{ Tarifstufe $1($, SNT“) } & \multicolumn{4}{|c|}{ Tarifstufe $3(, H T “)$} \\
\hline & \multicolumn{2}{|c|}{ Mo-Fr } & \multicolumn{2}{|l|}{ Sa/So } & \multicolumn{2}{|c|}{ Mo-Fr } & \multicolumn{2}{|l|}{$\mathrm{Sa} / \mathrm{So}$} \\
\hline & Sommer & Winter & Sommer & Winter & Sommer & Winter & Sommer & Winter \\
\hline 0 & $6,3 \%$ & $2,7 \%$ & $3,3 \%$ & $4,1 \%$ & $2,2 \%$ & $-3,3 \%$ & & \\
\hline 1 & $4,1 \%$ & $-0,9 \%$ & $-4,3 \%$ & $-1,1 \%$ & $-2,5 \%$ & $-4,2 \%$ & & \\
\hline 2 & $5,9 \%$ & $-0,4 \%$ & $7,2 \%$ & $0,0 \%$ & $-2,5 \%$ & $-5,0 \%$ & & \\
\hline 3 & $7,0 \%$ & $1,6 \%$ & $7,2 \%$ & $3,8 \%$ & $4,5 \%$ & $-3,2 \%$ & & \\
\hline 4 & $12,4 \%$ & $4,9 \%$ & $11,5 \%$ & $3,8 \%$ & $5,2 \%$ & $5,4 \%$ & & \\
\hline 5 & $11,5 \%$ & $3,5 \%$ & $6,2 \%$ & $2,4 \%$ & $6,1 \%$ & $-0,2 \%$ & & \\
\hline 6 & $3,4 \%$ & $-3,9 \%$ & $2,7 \%$ & $-0,3 \%$ & $-2,2 \%$ & $-6,4 \%$ & $1,7 \%$ & $0,5 \%$ \\
\hline 7 & $10,7 \%$ & $-2,9 \%$ & $3,5 \%$ & $5,4 \%$ & $-1,5 \%$ & $-9,6 \%$ & $-6,1 \%$ & $-5,8 \%$ \\
\hline 8 & $15,1 \%$ & $16,2 \%$ & $-1,6 \%$ & $4,3 \%$ & $-4,9 \%$ & $-3,5 \%$ & $-12,6 \%$ & $-13,1 \%$ \\
\hline 9 & $24,4 \%$ & $18,0 \%$ & $6,8 \%$ & $10,3 \%$ & $-1,1 \%$ & $-1,2 \%$ & $-11,7 \%$ & $-12,8 \%$ \\
\hline 10 & $13,0 \%$ & $15,1 \%$ & $0,5 \%$ & $7,0 \%$ & $-1,8 \%$ & $-1,8 \%$ & $-20,3 \%$ & $-15,2 \%$ \\
\hline 11 & $16,6 \%$ & $22,1 \%$ & $7,7 \%$ & $6,9 \%$ & $-2,4 \%$ & $-1,1 \%$ & $-11,6 \%$ & $-12,9 \%$ \\
\hline 12 & $11,0 \%$ & $15,8 \%$ & $4,7 \%$ & $3,3 \%$ & $-4,6 \%$ & $-4,4 \%$ & $-9,0 \%$ & $-13,0 \%$ \\
\hline 13 & $22,7 \%$ & $24,5 \%$ & $0,3 \%$ & $17,3 \%$ & $-2,5 \%$ & $-5,5 \%$ & $-4,5 \%$ & $-7,4 \%$ \\
\hline 14 & $13,8 \%$ & $23,3 \%$ & $10,0 \%$ & $10,7 \%$ & $-3,8 \%$ & $-5,6 \%$ & $-9,7 \%$ & $-8,5 \%$ \\
\hline 15 & $27,5 \%$ & $19,3 \%$ & $28,9 \%$ & $29,9 \%$ & $-1,9 \%$ & $-2,8 \%$ & $-12,0 \%$ & $-14,8 \%$ \\
\hline 16 & $23,7 \%$ & $18,3 \%$ & $14,7 \%$ & $15,6 \%$ & $2,9 \%$ & $-3,2 \%$ & $-10,5 \%$ & $-12,0 \%$ \\
\hline 17 & $22,0 \%$ & $5,0 \%$ & $11,2 \%$ & $11,9 \%$ & $1,3 \%$ & $-1,3 \%$ & $-2,9 \%$ & $-10,0 \%$ \\
\hline 18 & $12,1 \%$ & $2,8 \%$ & $8,8 \%$ & $2,1 \%$ & $2,7 \%$ & $-5,6 \%$ & $-0,2 \%$ & $-4,2 \%$ \\
\hline 19 & $13,0 \%$ & $5,1 \%$ & $-11,8 \%$ & $5,6 \%$ & $-0,1 \%$ & $-6,7 \%$ & $-2,2 \%$ & $-7,6 \%$ \\
\hline 20 & $-0,8 \%$ & $-6,8 \%$ & $7,0 \%$ & $2,6 \%$ & $-10,7 \%$ & $-13,0 \%$ & $-5,4 \%$ & $-9,4 \%$ \\
\hline 21 & $6,6 \%$ & $-2,5 \%$ & $3,1 \%$ & $9,1 \%$ & $-7,0 \%$ & $-11,3 \%$ & $-5,2 \%$ & $-7,1 \%$ \\
\hline 22 & $6,6 \%$ & $3,2 \%$ & $2,8 \%$ & $8,1 \%$ & $-4,1 \%$ & $-10,7 \%$ & & \\
\hline 23 & $9,0 \%$ & $5,0 \%$ & $6,3 \%$ & $6,3 \%$ & $0,8 \%$ & $-9,2 \%$ & & \\
\hline
\end{tabular}

Waren obige Ausführungen vor allem auf die Betrachtung der einzelnen Zeitscheiben fokussiert, erfolgte zusätzlich noch eine Untersuchung, inwieweit auch die Bepreisung der Vor- und Folgestunde das Lastverhalten beeinflussen. Exemplarisch sind in Abb. 8 die möglichen Tarifstufenkombinationen bei Gültigkeit der Tarifstufe 1 und 3 in der aktuellen Stunde aufgetragen. Es zeigt sich, dass die Lastverlagerungen bei Gültigkeit der Tarifstufe 1 je nach Tarifstufe in der Vor- und Folgestunde Schwankungen von bis zu 9 Prozentpunkten unterliegen. Die größten Lastverlagerungen werden überwiegend dann erzielt, wenn die günstigste Tarifstufe 1 in mindestens zwei aufeinanderfolgenden Zeitscheiben zur Anwendung gelangt. Demgegenüber sind bei Gültigkeit der hochpreisigen Tarifstufe 3 in der aktuellen Stunde Schwankungen von bis zu 6 Prozentpunkten auszumachen.

Berücksichtigt werden muss allerdings die Tatsache, dass die Anzahl der jeweiligen Tarifstufenkombinationen insgesamt sowie in den einzelnen Tagesabschnitten stark schwan- ken. Dies ist dem Umstand geschuldet, dass in den einzelnen Projektphasen im Rahmen der sog. Vertriebsregeln Zeitzonen definiert wurden, in denen jede Tarifstufe in einer vorbestimmten Anzahl aufzutreten hatte. Zum einen geschah dies, um den Signalverlauf für die Testkunden in gewissen Grenzen planbar gestalten zu können, um so die Teilnahmebereitschaft am Feldtest zu erhöhen, zum anderen konnte so zur Bestimmung der Kundenreaktionen ein Mindestmaßan täglichen Signalwechseln gewährleistet werden.

Tabelle 5 zeigt die Auftrittshäufigkeiten der in Abb. 8 aufgenommenen Tarifstufenkombinationen je Zeitzone. Die Zeitzonendefinition orientiert sich hierbei an den im Betrachtungszeitraum gültigen Vertriebsregeln, wobei die $\mathrm{Zu}$ ordnung des Messwertes anhand des mittleren Zeitsegmentes (,aktuelle Stunde“) erfolgte, also jener Zeitscheibe, auf die sich auch die in Abb. 8 angegebene Laständerung bezieht. Daher kann die der Tarifstufenkombination zu Grunde liegende Vor- oder Folgestunde auch in einer angrenzenden Zeitzone liegen. Mit Rücksicht auf eine Änderung der Ver- 


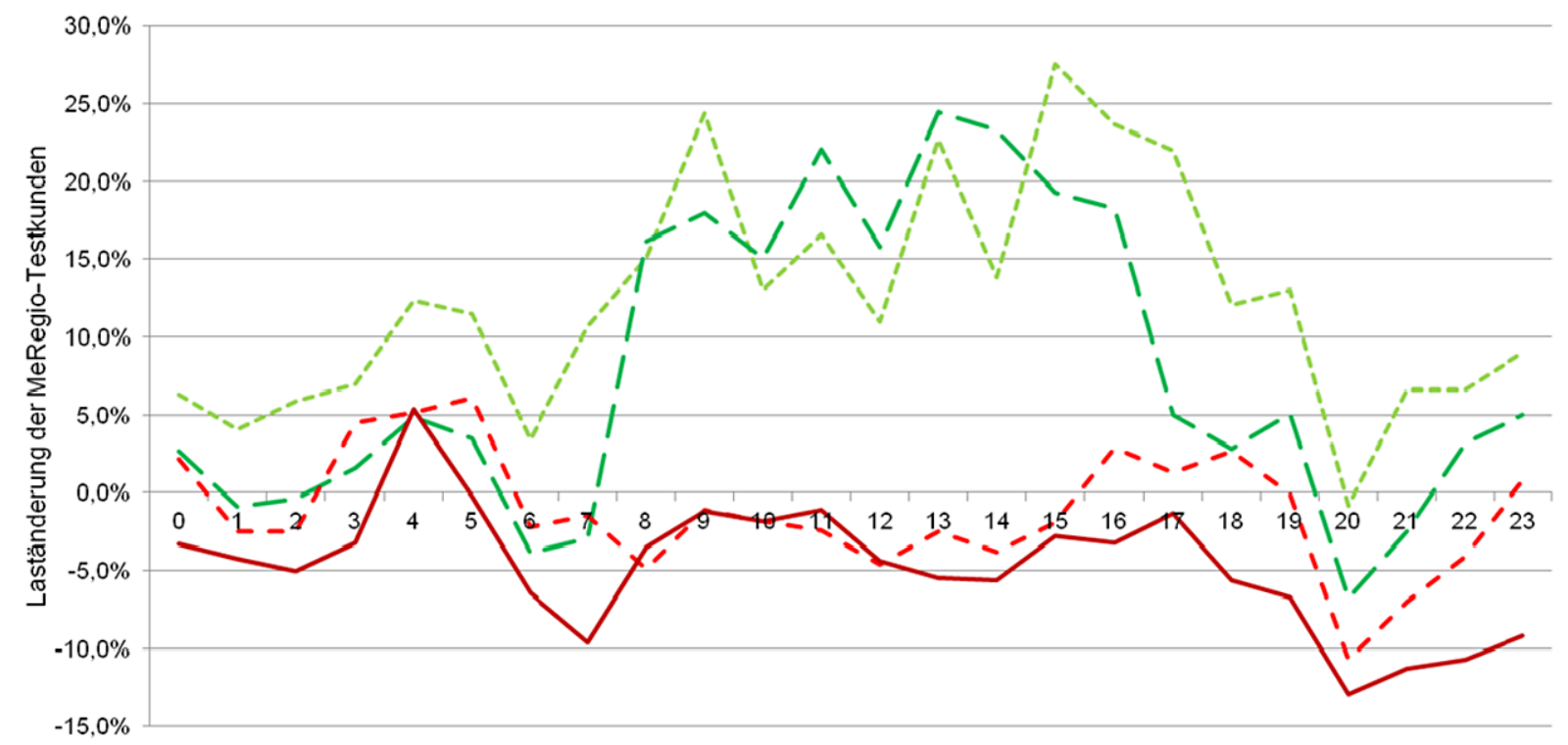

Zeit [h]

----Tarifstufe 1 Mo-Frim Sommer

- - Tarifstufe 3 Mo-Frim Sommer

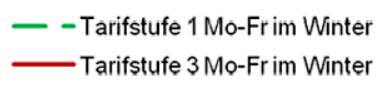

Abb. 6 Tageszeitabhängige relative Laständerung der MeRegio-Testkunden bezogen auf die Referenzgruppe montags bis freitags

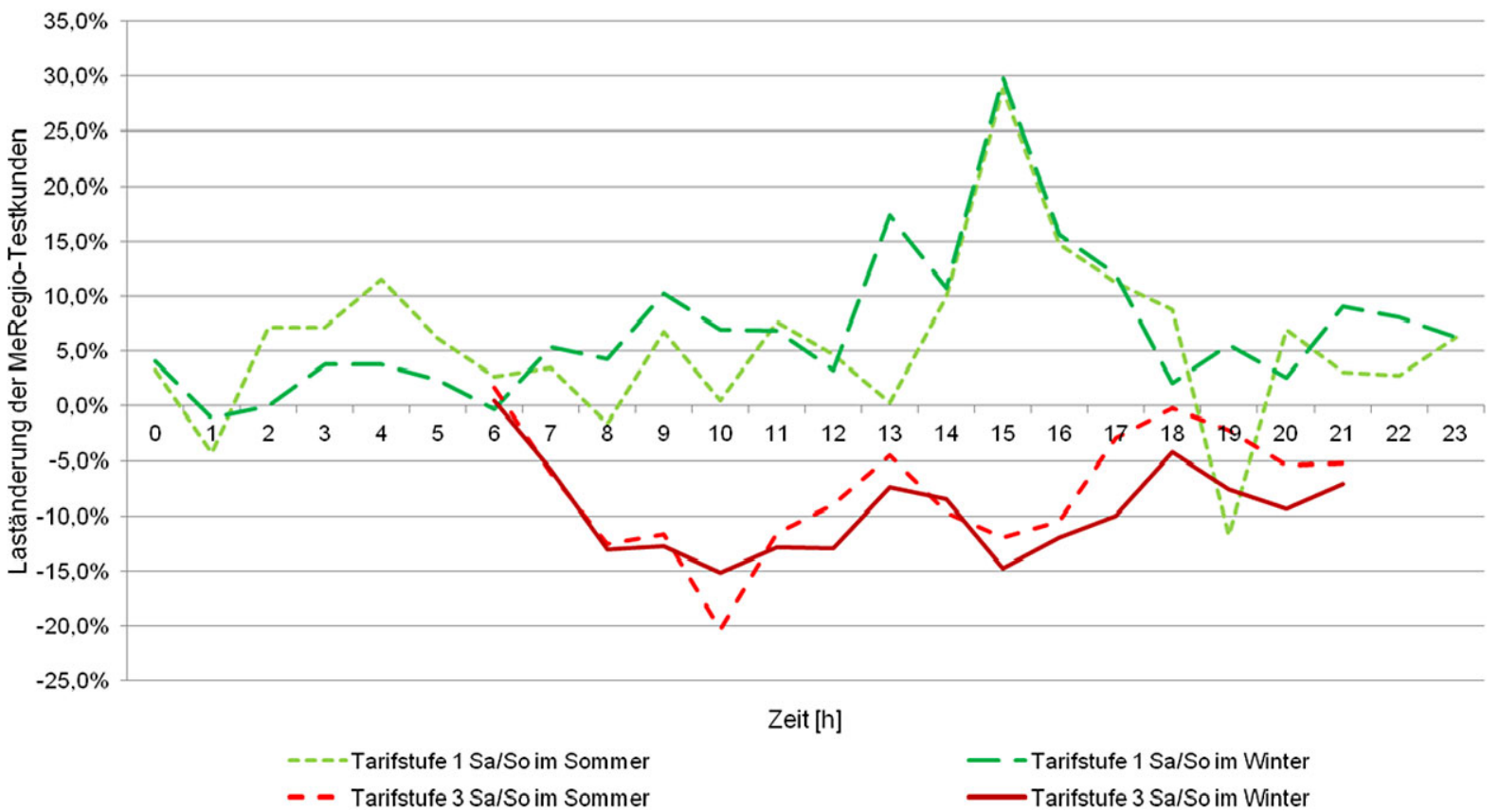

Abb. 7 Tageszeitabhängige relative Laständerung der MeRegio-Testkunden bezogen auf die Referenzgruppe am Wochenende sowie an Feiertagen

triebsregeln und damit einhergehend der Auftrittshäufigkeiten der Tarifkombinationen beschränkt sich die Betrachtung auf den Zeitraum von Beginn des Feldtests bis zum Januar 2011.

Aus Tab. 5 wird ersichtlich, dass beispielsweise ein dreistündiger Block der günstigsten Tarifstufe 1 (Kombination SNT-SNT-SNT) im Betrachtungszeitraum überwiegend in den Nachtstunden auftrat. Dieser Umstand erklärt, dass die mittlere relative Lastverlagerung mit 3,65 \% vergleichsweise gering ausfällt. Nimmt man zum Vergleich die Tarifstufenkombinationen, bei denen ein zweistündiger Block der günstigsten Tarifstufe auftritt und die gleichmäßiger über den Tag verteilt sind, stellt man eine deutlich größere Lastverlagerung fest, als wenn bei gleicher Verteilung über 


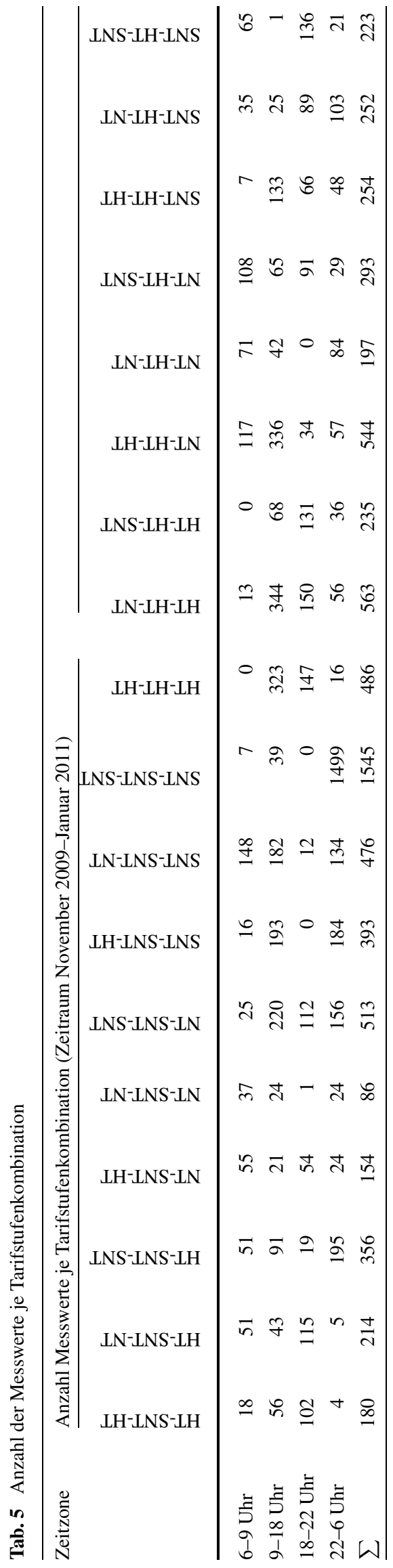

den Tag die günstigste Tarifstufe lediglich eine Stunde lang gilt.

Zusammenfassend lässt sich feststellen, dass die Sensitivitäten der Testkunden asymmetrisch ausgestaltet sind und es somit zur Lastverringerung nur eines vergleichsweise kurzzeitigen Anreizes in Form einer hochpreisigen Tarifstufe bedarf, zur Lasterhöhung dagegen ein länger andauernder Preisanreiz vonnöten ist. Dies lässt den Schluss zu, dass ein größerer Teil der Lastverlagerung über die Verschiebung planbarer, längerer Anwendungen (z. B. Waschen, Trocknen) geschieht, die in der Regel mehr als ein im Feldtest einstündiges Zeitsegment beanspruchen.

Insgesamt zeigen die Ergebnisse eindeutig, dass mit dem im Rahmen des Forschungsprojekts MeRegio aufgebauten System eine gezielte Lastbeeinflussung möglich ist. Dies auch, obwohl bislang das direkt steuernde Regelsignal in der untersuchten Datenbasis nicht berücksichtigt wurde. Es lässt sich somit festhalten, dass mittels wettbewerblicher Maßnahmen, namentlich einem Preissignal, Lastbeeinflussungen und Verhaltensanpassungen von Kunden erreicht werden können.

Das vorgeschlagene Rollenmodell wurde exemplarisch in einer Testumgebung erprobt, es entspricht der von der BNetzA vorgeschlagenen Rollenverteilung, bei der der Dynamische Bilanzkreisbewirtschafter dem Wettbewerbsbereich zugeordnet wird (BNetzA 2011). Da für dieses Rollenmodell damit nicht nur die grundsätzliche Funktionsfähigkeit nachgewiesen wurde, sondern es auch im Hinblick auf die Integration in das bestehende Regelwerk sowie die Kongruenz mit den Anforderungen und Wünschen der wichtigsten Stakeholder große Stärken zeigt, sollte es für die Weiterentwicklungen im Bereich Smart Grid/Smart Market sowie in zukünftig zu treffenden Regulierungsvorhaben bzw. Vorgaben für Standardprozesse zugrunde gelegt werden.

\section{Zusammenfassung und Ausblick}

Hervorgerufen durch den Umbruch im Energieversorgungssystem fällt auf Grund der Volatilität dezentraler Einspeisung einem intelligenten dezentralen Ausgleich zwischen Last und Erzeugung ein zunehmend stärkeres Gewicht zu. Dieser Ausgleich kann jedoch nur durch die Einbeziehung aller Marktakteure gelingen.

Ein zentraler Untersuchungsgegenstand des Forschungsprojektes MeRegio ist die Integration der Endkunden in diesen Ausgleichsprozess. Hierfür wurde ein Rollenmodell entwickelt, das im Einklang mit den Vorstellungen des Bundeswirtschaftsministeriums sowie der Bundesnetzagentur steht. Im Rahmen des Forschungsprojekts wurden diese Rollen und Prozesse implementiert und erfolgreich getestet.

Die Anreize für die Kunden erfolgen sowohl über Preisals auch über Regelsignale. Im Projekt konnte gezeigt wer- 

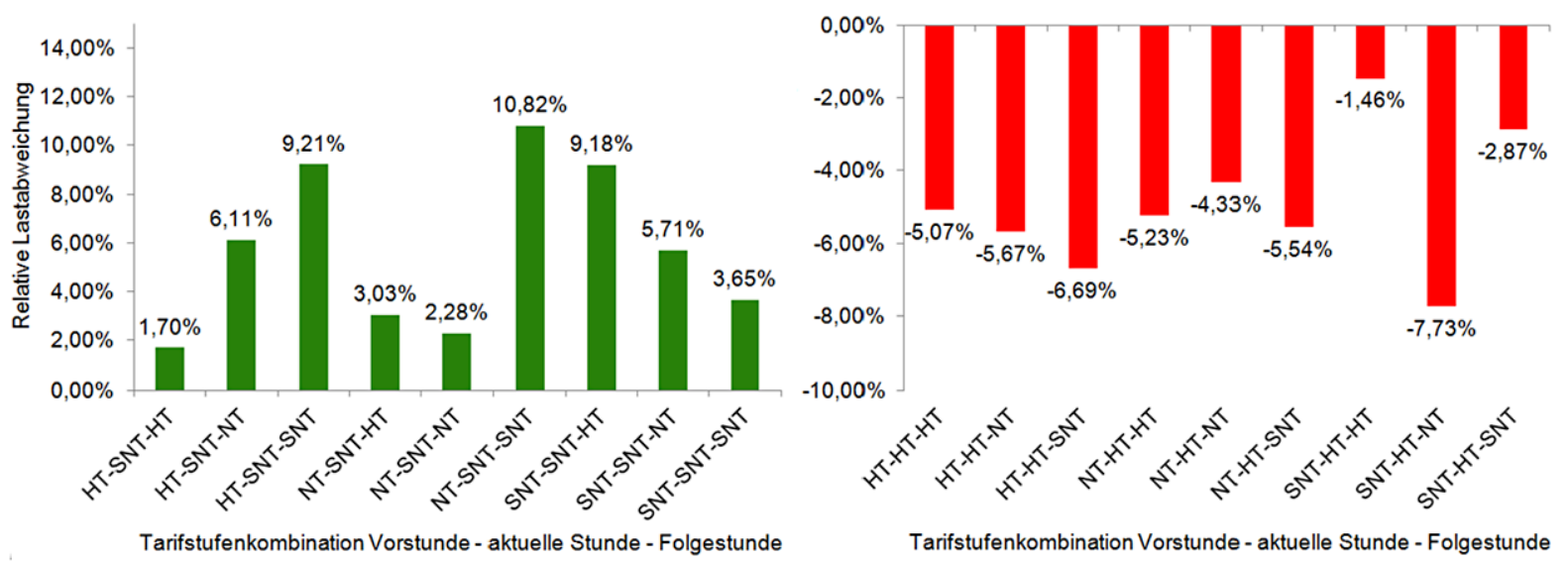

Abb. 8 Relative Laständerung der Testkunden in Abhängigkeit von der Tarifstufenkombination

den, dass die Endkunden eine deutliche Reaktion auf derartige Signale zeigen, allerdings ist die Reaktion abhängig vom Tagtyp, von der Jahres- und Tageszeit sowie von der Signalfolge. Diese Aspekte sind somit zu berücksichtigen, wenn ein Ausgleich der volatilen Erzeugung über einen Lastmanagementansatz erfolgen soll. Auch wenn es sich bei den Testkunden um einen kleinen Teilnehmerkreis handelt, der nicht notwendigerweise als repräsentativ anzusehen ist, da er sich freiwillig zur Versuchsteilnahme entschieden hat, sind die Ergebnisse dennoch ermutigend im Hinblick auf die Weiterentwicklung im Bereich Smart Market/Smart Grid. Weitere Auswertungen zur vertieften Analyse der Effekte erfolgen auch nach Ende des Feldtests.

Die Ergebnisse der ersten Auswertungen des Forschungsprojekts zeigen auch, dass das gewählte Rollenmodell geeignet ist, eine entsprechende Steuerung im dezentralen Bereich zu erreichen. Dabei weist das Modell speziell im Hinblick auf die Einbettung in das existierende Regulierungsund Rollenmodell als auch im Hinblick auf die Kundenakzeptanz Stärken auf, die im Rahmen der Weiterentwicklung der Thematik berücksichtigt werden sollten. Insbesondere konnte die Funktionsfähigkeit der anreizbasierten Laststeuerung durch Aussendung von Preissignalen aus dem wettbewerblichen Bereich nachgewiesen werden.

Da die Reaktionen der MeRegio-Testkunden auf die Anreizsignale noch überwiegend manuell erfolgen, ist anzunehmen, dass durch einen höheren Automatisierungsgrad und den damit verbundenen Komfortgewinn für die Kunden eine höhere Bereitschaft zur Teilnahme an entsprechenden Lastmanagement-Prozessen entstehen wird und somit zusätzliche Lastverlagerungspotenziale realisierbar wären. Darüber hinaus könnte ein vollständiges Erschließen der Flexibilität von Elektrowärmeanwendungen und zukünftiger Elektromobilität zu einer weiteren Steigerung des Lastverschiebepotenzials beitragen (Jochem et al. 2012).

\section{Literatur}

Bayley C, French S (2008) Designing a participatory process for stakeholder involvement in a societal decision. Group Decis Negot 17:195-210

Belton V, Stewart T (2002) Multiple criteria decision analysis-an integrated approach. Kluwer Academic, Boston

Bertsch V, Geldermann J (2008) Preference elicitation and sensitivity analysis in multi-criteria group decision support for industrial risk and emergency management. Int J Emerg Manag 5(1/2):7-24

Boiteux M (1949) La tarification des demands en point: application del la théorie de la vente au coût marginal. Rev Gen Électr 58:321-340; ins Englisch übertragen von H.W. Izzard als: PeakLoadPricing, The Journal of Business, 33, 157-179, 1960

Bose U, Davey AM, Olson DL (1997) Multi-attribute utility methods in group decision making: past applications and potential for inclusion in GDSS. Int J Manag Sci 25(6):691-706

Brauner G, Pöppl G, Tiefgraber D (2006) Verbraucher als virtuelles Kraftwerk. in: Berichte aus Energie- und Umweltforschung, $\mathrm{Nr} 44$

Bundesnetzagentur (2011) „Smart Grid“ und „Smart Market“: Eckpunktepapier der Bundesnetzagentur für Elektrizität, Gas, Telekommunikation, Post und Eisenbahnen zu den Aspekten des sich verändernden Energieversorgungssystems

Droste-Franke B, Paal B, Rehtanz C, Sauer D, Schneider J, Schreurs M, Ziesemer T (2012) Balancing renewable electricity: energy storage, demand side management and network extension from an interdisciplinary perspective. Springer, Berlin

Hillemacher L, Eßer-Frey A, Fichtner W (2011) Preis- und Regelsignale im MeRegio Smart Grid Feldtest - Simulationen und Erste Ergebnisse. In: Proceedings der 7. Internationalen Energiewirtschaftstagung, Wien

Hillemacher L, Jochem P, Fichtner W (2012) Entscheidungsunterstützung beim Lastmanagement. In: Renatus F, Kunze R, Karschin I, Geldermann J, Fichtner W (Hrsg) Entscheidungsunterstützung durch Operations Research im Energie- und Umweltbereich Tagungsband des Workshops der GOR-Arbeitsgruppen „OR im Umweltschutz“ \& „Entscheidungstheorie und Praxis“, 01. und 02. März 2012, Goslar, Aachen

Hirsch C, Hillemacher L, Block C, Schuller A, Möst D (2010) Simulations in the smart grid field study MeRegio. Inf Technol $\mathrm{J}$ 52(2):100-106

Hufendiek K, Wiechmann H (2012) Market roles and a system structure for smart Market/Grid — design and experiences from test region. Vertrag beim Enerday. In: 7th conference on energy economics and technology, Dresden, 27 April 2012 
Jochem P, Kaschub T, Paetz A-G, Fichtner W (2012) Integrating electric vehicles into the German electricity grid - an interdisciplinary analysis. In: EVS26, Los Angeles, USA

MeRegio-Projektkonsortium (2012) MeRegio - Aufbruch zu Minimum EmissionsRegions. Projekthomepage: www.meregio.de. Letztes Abrufdatum: 07.08.2012

Morovic T, Pilhar R, Möhring-Hüser W (1998) Dynamische Stromtarife und Lastmanagement - Erfahrungen und Perspektiven. Forschungsgesellschaft für umweltschonende Energieumwandlung und -nutzung $\mathrm{mbH}$, Kiel

Nabe C, Beyer C, Brodersen N, Schäffler H, Adam D, Heinemann C, Tusch T, Eder J, de Wyl C, vom Wege J-H, Mühe S (2010) Einführung von lastvariablen und zeitvariablen Tarifen. Studie im Auftrag der Bundesnetzagentur für Elektrizität, Gas, Telekommunikation, Post und Eisenbahnen
Nestle D (2007) Energiemanagement in der Niederspannungsversorgung mittels dezentraler Entscheidung. Dissertation, Universität Kassel

Oberschmidt J (2010) Multikriterielle Bewertung von Technologien zur Bereitstellung von Strom und Wärme. Dissertation, Universität Göttingen

Ockenfels A, Crampton P (2011) Is a capacity market required in Germany to guarantee system security? Report on two studies funded by RWE, Cologne, July 2011

Steiner PO (1957) Peak loads and efficient pricing. Q J Econ 71(4):585-610

von Winterfeldt D, Edwards W (1986) Decision analysis and behavioral research. Cambridge University Press, Cambridge 


\section{Repository KITopen}

Dies ist ein Postprint/begutachtetes Manuskript.

Empfohlene Zitierung:

Hillemacher, L.; Hufendiek, K.; Bertsch, V.; Wiechmann, H.; Gratenau, J.; Jochem, P.; Fichtner, W.

Ein Rollenmodell zur Einbindung der Endkunden in eine smarte Energiewelt. 2013. Zeitschrift für Energiewirtschaft, 37. doi: $10.554 / / R / 1000045253$

Zitierung der Originalveröffentlichung:

Hillemacher, L.; Hufendiek, K.; Bertsch, V.; Wiechmann, H.; Gratenau, J.; Jochem, P.; Fichtner, W.

Ein Rollenmodell zur Einbindung der Endkunden in eine smarte Energiewelt. 2013. Zeitschrift für Energiewirtschaft, 37 (3), 195-210.

doi:10.1007/s12398-013-0110-z 